\title{
Observing the First Stars and Black Holes
}

\author{
Zoltán Haiman \\ Department of Astronomy, Columbia University, New York, NY 10027 \\ zoltan@astro.columbia.edu
}

Summary. The high sensitivity of JWST will open a new window on the end of the cosmological dark ages. Small stellar clusters, with a stellar mass of several $\times 10^{6} \mathrm{M}_{\odot}$, and low-mass black holes (BHs), with a mass of several $\times 10^{5} \mathrm{M}_{\odot}$ should be directly detectable out to redshift $z=10$, and individual supernovae ( $\mathrm{SNe}$ ) and gamma ray burst GRB afterglows are bright enough to be visible beyond this redshift. Dense primordial gas, in the process of collapsing from large scales to form protogalaxies, may also be possible to image through diffuse recombination line emission, possibly even before stars or BHs are formed. In this article, I discuss the key physical processes that are expected to have determined the sizes of the first star-clusters and black holes, and the prospect of studying these objects by direct detections with JWST and with other instruments. The direct light emitted by the very first stellar clusters and intermediate-mass black holes at $z>10$ will likely fall below JWST's detection threshold. However, JWST could reveal a decline at the faint-end of the high-redshift luminosity function, and thereby shed light on radiative and other feedback effects that operate at these early epochs. JWST will also have the sensitivity to detect individual $\mathrm{SNe}$ from beyond $z=10$. In a dedicated survey lasting for several weeks, thousands of $\mathrm{SNe}$ could be detected at $z>6$, with a redshift distribution extending to the formation of the very first stars at $z \gtrsim 15$. Using these SNe as tracers may be the only method to map out the earliest stages of the cosmic starformation history. Finally, we point out that studying the earliest objects at high redshift will also offer a new window on the primordial power spectrum, on $\sim 100$ times smaller scales than probed by current large-scale structure data.

\section{Introduction}

The formation of the first astrophysical objects and the subsequent epoch of reionization are at the frontiers of research in Astronomy. Recent years have seen significant progress both in our theoretical understanding and in observational probes of this transition epoch in the early universe. Through a combination of methods, including measurements of the cosmic microwave background (CMB) anisotropies, culminating in the precise determination of the temperature and polarization power spectra from the Wilkinson Microwave Anisotropy Probe (WMAP) experiment [16, 159, 160, 134, 42, 96], Hubble diagrams of distant Supernovae [139 138], and various probes of large scale structure (see, e.g., references in [5 172] 156]) the key cosmological parameters have been determined to high accuracy. 
Because of the emergence of a concordance $(\Lambda \mathrm{CDM})$ cosmology, we can securely predict the collapse redshifts of the first non-linear dark matter condensations: $2-3 \sigma$ peaks of the primordial density field on mass scales of $10^{5-6} \mathrm{M}_{\odot}$, corresponding to the cosmological Jeans mass, collapse at redshifts $z=15-20$. Of course, in addition to the cosmological parameters describing the average background universe $(\Lambda \mathrm{CDM})$, one also needs a description of the seed fluctuations, to make such predictions. The nearly scale-invariant nature of the initial fluctuation power spectrum has also been empirically confirmed by the above-mentioned large-scale structure data. However, the predictions for the earliest halos rely on extrapolating the primordial power spectrum to a wavenumber of $k \sim 10 \mathrm{~h} \mathrm{Mpc}^{-1}$, a scale that is $2-3$ orders of magnitude smaller than the smallest scale directly probed by current data (e.g. [178] and references therein). It is possible that the small-scale power differs substantially from the extrapolated value. For example, in the case of warm dark matter (WDM), the power can be reduced by many orders of magnitude on the relevant scales, and, to zeroth order, the first generation of halos predicted in $\Lambda \mathrm{CDM}$ would not exist [6]. Recent simulations indicate that the formation of the first halos would indeed be delayed [132, 193], and the details of the collapse dynamics modified, possibly ultimately affecting the properties of the first stars [61]. Within the $\Lambda \mathrm{CDM}$ paradigm, however, robust predictions can be made, using three-dimensional simulations [195, 162], rather than semi-analytical methods [137] 154]. Such predictions are now limited mainly by the $\approx 5 \%$ uncertainty in the normalization of the primordial power spectrum, $\sigma_{8 h^{-1}}$ (e.g. [156]), which translates essentially to a $\approx 5 \%$ uncertainty in the collapse redshift of the first structures.

On the observational side, the recent measurement of the optical depth to electron scattering $\left(\tau_{e}=0.09 \pm 0.03\right)$ by WMAP suggests that the first sources of light significantly ionized the intergalactic medium (IGM) at redshift $z \sim 11 \pm 3$ [134, 160]. The Sloan Digital Sky Survey (SDSS) has uncovered a handful of bright quasars at redshifts as high as $z=6.41$ (see [14, 46, 46, 47, 48, 49] or the recent review by [50]). The luminosity of these sources rivals those of the most powerful quasars at the peak of their activity at $z \simeq 2.5$. A sizable population of $z \sim 6$ galaxies have also been identified in the past several years (see, e.g., [44] for a recent comprehensive review), many of them in broad-band photometry in deep, wide-area Hubble Space Telescope fields [35, 112, 19, 164, 165]. Narrow-band searches have also been successful in discovering numerous $z>5$ Lyman $-\alpha$ emitting galaxies. Starting with the initial success of [182], 10m class telescopes have now identified about two dozen such objects out to $z \approx 6.7$ (see [111] for a compilation of datasets; and the more recent Subaru survey results by, e.g., [169, 93]). In summary, the above observational data leave little doubt that the first galaxies and quasars were indeed well in place by redshift $z \simeq 6$, but not in significant numbers prior to redshift $z \simeq 15$.

These developments have been complemented by progress in theoretical work focusing on the cooling and collapse of gas in primordial low-mass halos (see reviews by [71,2] and references therein). A broad picture has emerged, identifying several processes that are important in the formation of the first stars and black holes (BHs) out of the baryonic gas in the earliest halos. The key ingredient in this picture is the abundance of $\mathrm{H}_{2}$ molecules that form via gasphase reactions in the early universe (this beautifully simple fact was recognized as early as in 1967 by [147]). The first objects form out of gas that cools via $\mathrm{H}_{2}$ molecules, and condenses at the centers of virialized dark matter "minihalos" with virial temperatures of $T_{\text {vir }} \sim 200 \mathrm{~K}$ [82, 171]. Detailed numerical simulations have shown convergence toward a gas temperature $T \sim 300 \mathrm{~K}$ and density $n \sim 10^{4} \mathrm{~cm}^{-3}$, dictated by the thermodynamic properties of $\mathrm{H}_{2}$ [1, 20, 192, 194], which allows the collapse of a single clump of mass $10^{2}-10^{3} \mathrm{M}_{\odot}$ at the center of the halo. The soft UV and X-ray radiation emitted by the stars and black holes formed in the first handful of such clumps provide prompt and significant global feedback on the chem- 
ical and thermal state of the IGM. A relatively feeble early background radiation field can already have strong effects on gas cooling and $\mathrm{H}_{2}$ chemistry, and can produce significant global impact, affecting the formation of the bulk of the first astrophysical structures, and therefore the reionization history of the IGM [80, 72, 106, 107, 129, 142, 97, 58, 116, 4, 91]. The observational data summarized above suggests that such feedback processes indeed shaped the reionization history, and hints that star-formation in early minihalos was suppressed [73].

\section{Summary of Relevant Physics at High Redshifts}

In the context of $\Lambda \mathrm{CDM}$ cosmologies, it is natural to identify the first dark matter halos as the hosts of the first sources of light. A simplified picture for the emergence of the first sources of light, and consequent reionization is as follows: the gas in dark matter halos cools and turns into ionizing sources (stars and/or black holes), which produce UV (and possibly $\mathrm{X}$ ray) radiation, and drive expanding ionized regions into the IGM. The volume filling factor of ionized regions then, at least initially, roughly tracks the global formation rate of dark halos. Eventually, the ionized regions percolate (when the filling factor $F_{\mathrm{HII}}$ reaches unity), and the remaining neutral hydrogen in the IGM is rapidly cleared away as the ionizing background builds up. However, a soft UV radiation background at photon energies of $<13.6 \mathrm{eV}$, as well as possibly a soft $\mathrm{X}$-ray background at $\gtrsim 1 \mathrm{keV}$, will build up before reionization, since the early IGM is optically thin at these energies.

We now briefly describe the various effects that should determine the evolution of the first sources and of reionization at high redshifts. For in-depth discussion, we refer the reader to extended reviews (e.g. [9]), and to reviews focusing on the roles of $\mathrm{H}_{2}$ molecules in reionization [2], on the effect of reionization on CMB anisotropies [75], and on progress in the last three years in studying reionization [71, 54].

In the discussions that follow, it will be useful to distinguish three different types of dark matter halos, which can be roughly divided into three different ranges of virial temperatures, as follows:

$$
\begin{aligned}
& 300 \mathrm{~K} \lesssim T_{\text {vir }} \lesssim 10^{4} \mathrm{~K} \quad \text { (Type II - susceptible to } \mathrm{H}_{2} \text {-feedback) } \\
& 10^{4} \mathrm{~K} \lesssim T_{\text {vir }} \lesssim 2 \times 10^{5} \mathrm{~K} \quad \text { (Type Ia - susceptible to photo-heating feedback) } \\
& T_{\text {vir }} \gtrsim 2 \times 10^{5} \mathrm{~K} \quad \text { (Type } \mathrm{Ib}-\text { gas can fall in, even in the face of photo-heating) }
\end{aligned}
$$

We will hereafter refer to these three different types of halos as Type II, Type Ia, and Type Ib halos. The motivation in distinguishing Type II and Type I halos is based on $\mathrm{H}_{2}$ molecular vs atomic $\mathrm{H}$ cooling, whereas types "a" and "b" reflect the ability of halos to allow infall and cooling of photoionized gas. While the actual values of the critical temperatures, and the sharpness of the transition from one category to the next is uncertain (see discussion below), as we argue below, star formation in each type of halo is likely governed by different physics, and each type of halo likely plays a different role in the reionization history. In short, Type II halos can host ionizing sources only in the neutral regions of the IGM, and only if $\mathrm{H}_{2}$ molecules are present; Type Ia halos can form new ionizing sources only in the neutral IGM regions, but can do so by atomic cooling, irrespective of the $\mathrm{H}_{2}$ abundance, and Type Ib halos can form ionizing sources regardless of the $\mathrm{H}_{2}$ abundance, and whether they are in the ionized or neutral phase of the IGM. It is useful to next provide a summary of the various important physical effects that govern star-formation and feedback in these halos. 
Table 1. Collapse redshifts and masses of 1,2 and $3 \sigma$ dark matter halos with virial temperatures of $T_{\mathrm{vir}}=100$ and $10^{4} \mathrm{~K}$, reflecting the minimum gas temperatures required for cooling by $\mathrm{H}_{2}$ and neutral atomic $\mathrm{H}$, respectively.

\begin{tabular}{lrrr}
$T_{\text {vir }}(\mathrm{K})$ & $v$ & $z_{\text {coll }}$ & $M_{\text {halo }}\left(\mathrm{M}_{\odot}\right)$ \\
\hline 100 & 1 & 7 & $2 \times 10^{5}$ \\
& 2 & 16 & $5 \times 10^{4}$ \\
& 3 & 26 & $3 \times 10^{4}$ \\
$10^{4}$ & 1 & 3.5 & $4 \times 10^{8}$ \\
& 2 & 9 & $1 \times 10^{8}$ \\
& 3 & 15 & $6 \times 10^{7}$ \\
\hline
\end{tabular}

$\mathrm{H}_{2}$ Molecule Formation. In $\Lambda \mathrm{CDM}$ cosmologies, structure formation is bottom-up: the earliest nonlinear dark matter halos form at low masses. Gas contracts together with the dark matter only in dark halos above the cosmological Jeans mass, $M_{\mathrm{J}} \approx 10^{4}$. However, this gas can only cool and contract to high densities in somewhat more massive halos, with $M \gtrsim M_{\mathrm{H} 2} \equiv 10^{5} M_{\odot}[(1+z) / 11]^{-3 / 2}$ (i.e. Type II halos), and only provided that there is a sufficient abundance of $\mathrm{H}_{2}$ molecules, with a relative number fraction at least $n_{\mathrm{H} 2} / n_{\mathrm{H}} \sim 10^{-3}$ [82, 171]. Because the typical collapse redshift of halos is a strong function of their size, the abundance of $\mathrm{H}_{2}$ molecules is potentially the most important parameter in determining the onset of reionization. For example, $2 \sigma$ halos with virial temperatures of $100 \mathrm{~K}$ appear at $z=16$, while $2 \sigma$ halos with virial temperatures of $10^{4} \mathrm{~K}$ (Type Ia halos, in which gas cooling is enabled by atomic hydrogen lines) appear only at $z=9$. Table 1 summarizes the collapse redshifts and masses of dark halos at these two virial temperatures in the concordance cosmology. As a result, the presence or absence of $\mathrm{H}_{2}$ in Type II halos makes a factor of $\sim 2$ difference in the redshift for the onset of structure formation, and thus potentially effects the reionization redshift and the electron scattering opacity $\tau$ by a similar factor. In the absence of any feedback processes, the gas collecting in Type II halos is expected to be able to form the requisite amount of $\mathrm{H}_{2}$ [82, 171]. However, both internal and external feedback processes can alter the typical $\mathrm{H}_{2}$ abundance (see discussion below).

The Nature of the Light Sources in the First Halos. A significant uncertainty is the nature of the ionizing sources turning on inside halos collapsing at the highest redshifts. Three dimensional simulations using adaptive mesh refinement (AMR; [1]) and smooth particle hydrodynamics (SPH; [20, 194]) techniques have followed the contraction of gas in Type II halos at high redshifts to high densities. These works have shown convergence toward a temperature/density regime of $\mathrm{T} \sim 200 \mathrm{~K}, \mathrm{n} \sim 10^{4} \mathrm{~cm}^{-3}$, dictated by the critical density at which the excited states of $\mathrm{H}_{2}$ reach equilibrium population levels. These results have suggested that the first Type II halos can only form unusually massive stars, with masses of at least $\sim 100 \mathrm{M}_{\odot}$, and only from a small fraction $(\lesssim 0.01)$ of the available gas. Such massive stars are effective producers of ionizing radiation, making reionization easier to achieve. In addition, it is expected that the first massive stars, forming out of metal-free gas, have unusually hard spectra [176, 22] 148], which is important for possibly ionizing helium, in addition to hydrogen. An alternative possibility is that a similar fraction of the gas in the first Type II halos forms massive black holes [76, 110, 144]. In fact, when massive, non-rotating metal-free stars end 
their life, they are expected to leave behind stellar-mass seed BHs, unless their mass is in the range of 140-260 $\mathrm{M}_{\odot}$ [84]. These early black holes can then accrete gas (possibly only after a delay - if the progenitor star clears the host halo of gas, the BH will have to await until the host halo merges with another, gas-rich halo), acting as "miniquasars". The miniquasars will produce a hard spectrum extending to the soft X-rays, which could be important in catalyzing $\mathrm{H}_{2}$ formation globally (see discussion below).

Efficiencies and the Transition from Metal Free to "Normal" Stars. Another fundamental question of interest is the efficiency at which the first sources inject ionizing photons into the IGM. This can be parameterized by the product $\varepsilon_{*} \equiv N_{\gamma} f_{*} f_{\mathrm{esc}}$, where $f_{*} \equiv M_{*} /\left(\Omega_{\mathrm{b}} M_{\text {halo }} / \Omega_{m}\right)$ is the fraction of baryons in the halo that turns into stars; $N_{\gamma}$ is the mean number of ionizing photons produced by an atom cycled through stars, averaged over the initial mass function (IMF) of the stars; and $f_{\text {esc }}$ is the fraction of these ionizing photons that escapes into the IGM. It is difficult to estimate these quantities at high-redshifts from first principles, but the discussion below can serve as a useful guide.

Although the majority of the baryonic mass in the local universe has been turned into stars [56], the global star formation efficiency at high redshifts was likely lower. To explain a universal carbon enrichment of the IGM to a level of $10^{-2}-10^{-3} \mathrm{Z}_{\odot}$, the required efficiency, averaged over all halos at $z \gtrsim 4$, is $f_{*}=2-20 \%$ [76]. However, the numerical simulations mentioned in $\S 2.2$ above suggest that the fraction of gas turned into massive stars in Type II halos is $f_{*} \lesssim 1 \%$.

The escape fraction of ionizing radiation in local starburst galaxies is of order $\sim 10 \%$. The higher characteristic densities at higher redshifts could decrease this value [41, 187], although there are empirical indications that the escape fraction in $z \sim 3$ galaxies may instead be higher [166], at least in some galaxies [153]. Radiation can also more readily ionize the local gas, and escape from the small Type II halos which have relatively low total hydrogen column densities $\left(\lesssim 10^{17} \mathrm{~cm}^{-2}\right.$ ), effectively with $f_{\text {esc }}=1$ in the smallest halos [183].

The ionizing photon yield per proton for a normal Salpeter IMF is $N_{\gamma} \approx 4000$. However, if the IMF consists exclusively of massive $M \gtrsim 200 \mathrm{M}_{\odot}$ metal-free stars, then $N_{\gamma}$ can be up to a factor of $\sim 20$ higher [22, 148]. The transition from metal-free to a "normal" stellar population is thought to occur at a critical metallicity of $Z_{\mathrm{cr}} \sim 5 \times 10^{-4} \mathrm{Z}_{\odot}$, above which cooling and fragmentation becomes efficient and stops the IMF from being biased toward massive stars [22]. It is natural to associate this transition with that of the assembly of halos with virial temperatures of $>10^{4} \mathrm{~K}$ (Type Ia halos). Type II halos are fragile, and likely blow away their gas and "shut themselves off" after a single episode of (metal-free) star-formation. They are therefore unlikely to allow continued formation of stars with metallicities above $Z_{\text {crit }}$. Subsequent star-formation will then occur only when the deeper potential wells of Type Ia halos are assembled and cool their gas via atomic hydrogen lines. The material that collects in these halos will then have already gone through a Type II halo phase and contain traces of metals.

As we argue in $\S 3$ below, there exists an alternative, equally plausible scenario. Most Type II halos may not have formed any stars, due to global $\mathrm{H}_{2}$ photodissociation by an early cosmic soft-UV background. In this case, the first generation of metal-free stars must appear in Type Ia halos. Halos above this threshold can eject most of their self-produced metals into the IGM, but, in difference from Type II halos, can retain most of their gas [108], and can have significant episodes of metal-free star formation. These halos will also start the process of reionization by driving expanding ionization fronts into the IGM. The metals that are ejected from Type Ia halos will reside in these photoionized regions of the IGM. As discussed in $\S 3$ below, photoionization heating in these regions may suppress gas infall and cooling, causing a pause in the formation of new structures, until larger dark matter halos, with virial tempera- 
tures of $T_{\text {vir }} \gtrsim 2 \times 10^{5} \mathrm{~K}$ (Type Ib halos) are assembled. The material that collects in Type Ib halos will then have already gone through a previous phase of metal-enrichment by Type Ia halos, and it is unlikely that Type Ib halos can form significant numbers of metal-free stars.

\section{Feedback Effects}

Several feedback effects are likely to be important for modulating the evolution of the early cosmic star formation history and reionization. There can be significant internal feedback in or near each ionizing source, due to the presence of supernovae [53], or of the radiation field [131, 142], on the local $\mathrm{H}_{2}$ chemistry. The net sign of these effects is difficult compute, as it depends on the source properties and spectra, and on the detailed density distribution internal and near to the sources. For practical purposes of computing the feedback that modulates the global star-formation or reionization, we may, however, think of any internal feedback effect as regulating the efficiency parameter $\varepsilon_{*}$ defined above.

Since the universe is optically thin at soft UV (below $13.6 \mathrm{eV}$ ), and soft X-ray ( $\gtrsim 1 \mathrm{keV})$ photon energies, radiation from the earliest Type II halos can build up global backgrounds at these energies, and provide prompt external, global feedback on the formation of subsequent structures, which are easier to follow. A very large number of studies over the past several years have assessed the feedback from both the LW and X-ray backgrounds quantitatively, including many works that employed three-dimensional simulations [72, 32, 106, 107, 142 143, 65, 97, 58, 116, 4, 91]. In isolation, Type II halos with virial temperatures as low as a few $100 \mathrm{~K}$ could form enough $\mathrm{H}_{2}$, via gas-phase chemistry, for efficient cooling and gas contraction [82 171]. However, $\mathrm{H}_{2}$ molecules are fragile, and can be dissociated by soft UV radiation absorbed in their Lyman-Werner (LW) bands [80, 32, 72, 141]. In patches of the IGM corresponding to fossil HII regions that have recombined (after the death of short-lived ionizing source, such as a massive star), the gas retains excess entropy for a Hubble time. This "entropy floor" can reduce gas densities in the cores of collapsing halos (analogously to the case of "preheating" of nearby galaxy clusters), and decrease the critical LW background flux that will photodissociate $\mathrm{H}_{2}$ [129]. On the other hand, positive feedback effects, such as the presence of extra free electrons (beyond the residual electrons from the recombination epoch) from protogalactic shocks [152, 53], from a previous ionization epoch [129, 168], or from $\mathrm{X}$-rays [80, 127, 142, 143], can enhance the $\mathrm{H}_{2}$ abundance.

The extent to which star-formation in Type II halos was quenched globally has remained unclear, with numerical simulations generally favoring less quenching [106 107 142] 143 186 than predicted in semi-analytical models. Likewise, simulations find a smaller, if any, effect from X-rays [97]. In a recent study [116], we used three-dimensional hydrodynamic simulations to investigate the effects of a transient ultraviolet (UV) flux and a LW background on the collapse and cooling of pregalactic clouds, with masses in the range $10^{5}-10^{7} \mathrm{M}_{\odot}$, at high redshifts $(z \gtrsim 18)$. In the absence of a LW background, we found that a critical specific intensity of $J_{\mathrm{UV}} \sim 0.1$ (in units of $10^{-21} \mathrm{ergs} \mathrm{s}^{-1} \mathrm{~cm}^{-2} \mathrm{~Hz}^{-1} \mathrm{sr}^{-1}$ ) demarcates the transition from net negative to positive feedback for the halo population. Note that this flux is $\sim 2$ orders of magnitude below the level required for reionization (defined by the requirement of producing a few photons per $\mathrm{H}$ atom). A weaker $\mathrm{UV}$ flux stimulates subsequent star formation inside the fossil HII regions, by enhancing the $\mathrm{H}_{2}$ molecule abundance. A stronger UV flux significantly delays star-formation by reducing the gas density, and increasing the cooling time at the centers of collapsing halos. At a fixed $J_{\mathrm{UV}}$, the sign of the feedback also depends strongly on the density of the gas at the time of UV illumination. In either case, once the UV flux is turned 
off, its impact starts to diminish after $\sim 30 \%$ of the Hubble time. In the more realistic case when a permanent LW background is present (in addition to a short-lived ionizing neighbor), with $J_{\mathrm{LW}} \gtrsim 0.01 \times 10^{-21} \mathrm{ergs} \mathrm{s}{ }^{-1} \mathrm{~cm}^{-2} \mathrm{~Hz}^{-1} \mathrm{sr}^{-1}$, strong suppression persists down to the lowest redshift $(z=18)$ in these simulations. The feedback was also found to depend strongly on the mass of the Type II halo, with the smaller halos strongly suppressed, but the larger halos $\left(\gtrsim 10^{7} \mathrm{M}_{\odot}\right)$ nearly immune to feedback. In recent works, [186] and [133] found that the largest Type II halos, with $T_{\text {vir }} \gtrsim 4000 \mathrm{~K}$, can eventually cool their gas even in the face of a LW background with $J_{\mathrm{LW}}>0.1$, due to the increased electron abundance and elevated temperature and cooling rate in the inner regions of these halos.

A second type of important feedback is that photo-ionized regions are photo-heated to a temperature of $\gtrsim 10^{4} \mathrm{~K}$, with a corresponding increase in the Jeans mass in these regions, and possible suppression of gas accretion onto low-mass halos (e.g. [43, 173, 67, 150]). Reionization is then expected to be accompanied by a drop in the global SFR, corresponding to a suppression of star formation in small halos (i.e. those with virial temperatures below $T_{\mathrm{vir}} \lesssim 10^{4}$ $-10^{5} \mathrm{~K}$ ). The size of such a drop is uncertain, since the ability of halos to self-shield against the ionizing radiation is poorly constrained at high redshifts. Early work on this subject, in the context of dwarf galaxies at lower redshift [173], suggested that an ionizing background would completely suppress star formation in "dwarf galaxy" halos with circular velocities $v_{\text {circ }} \lesssim 35 \mathrm{~km} \mathrm{~s}^{-1}$, and partially suppress star-formation in halos with $35 \mathrm{~km} \mathrm{~s}^{-1} \lesssim v_{\text {circ }} \lesssim$ $100 \mathrm{~km} \mathrm{~s}^{-1}$. However, more recent studies [95 36] find that at high-redshifts $(z \gtrsim 3)$, selfshielding and increased cooling efficiency could be strong countering effects. These calculations, however, assume spherical symmetry, leaving open the possibility of strong feedback for a halo with non-isotropic gas profile, illuminated along a low-column density line of sight (see [151] for a detailed treatment of three-dimensional gas dynamics in photo-heated lowmass halos).

Because the earliest ionizing sources formed at the locations of the rare density peaks, their spatial distribution was strongly clustered. Since most feedback mechanisms operate over a limited length scale, their effects will depend strongly on the spatial distribution of halos hosting ionizing sources. Numerical simulations are a promising way to address feedback among clustered sources, since they capture the full, three-dimensional relationships among the host halos [89]. However, the dynamic range required to resolve the small minihalos, within a large enough cosmic volume to be representative, remains a challenge, especially in simulations that include radiative transfer [88]. Semi-analytical models avoid the problems associated with the large dynamical range; they are also an efficient way to explore parameter space and serve as important sanity checks for more complicated simulations. Semi-analytical studies to date have included either various feedback effects (e.g. [77, 74, 189, 28, 70, 58 188. 91]) or the effect of source clustering on the HII bubble-size distribution (e.g. [59]), but not both. In a recent study [98], we have incorporated photo-ionization feedback, in a simplified way, into a model that partially captures the source clustering (i.e., only in the radial direction away from sources). Source clustering was found to increase the mean HII bubble size by a factor of several, and to dramatically increase the fraction of minihalos that are suppressed, by a factor of up to $\sim 60$ relative to a randomly distributed population. We argue that source clustering is likely to similarly boost the importance of a variety of other feedback mechanisms. (This enhanced suppression can also help reduce the electron scattering optical depth $\tau_{e}$, as required by the three-year data from WMAP [73].) 


\section{How can we detect this feedback?}

There are several ways, at least in principle, to discover the presence of global feedback mechanisms that modulate the early star-formation rate and reionization. Here I simply list several possibilities, in order to give an (admittedly crude) overview. In $\S 6$ I will discuss in more detail the one most likely to be relevant to JWST - tracing the cosmic star-formation history with $\mathrm{SNe}$, and searching for a feature in this ultra-high redshift version of the "Lilly-Madau" diagram, caused by the feedback.

In general, the feedback processes discussed above can produce an extended and complex (possibly even non-monotonic) reionization history, especially at the earliest epochs. The earliest stages of the global reionization history (ionized fraction versus cosmic time) can be probed in $21 \mathrm{~cm}$ studies (see [57] and the contributions by Steve Furlanetto and Avi Loeb in these proceedings). Additionally, the measurement of polarization anisotropies by Planck can go beyond a constraint on the total electron scattering optical depth $\tau_{e}$ that is measured by WMAP, and at least distinguish reionization histories that differ significantly [92 74,125 from each other. Reionization may modify the small-angle CMB anisotropies, as well, through the kinetic SZ effect, at a level that may be detectable in the future [145, 115, 114, 90, 146].

A recent study [123] considered the pumping of the $63.2 \mu \mathrm{m}$ fine-structure line of neutral oxygen in the high-redshift intergalactic medium (IGM), in analogy with the WouthuysenField effect for the $21 \mathrm{~cm}$ line of cosmic HI. This showed that the soft UV background at $1300 \AA$ can affect the fine-structure population levels in the ground state of OI. If a significant fraction of the IGM volume is filled with "fossil H II regions" that have recombined and contain neutral OI, then this can produce a non-negligible spectral distortion in the cosmic microwave background (CMB). A measurement of this signature can trace the global metallicity at the end of the dark ages, prior to the completion of cosmic reionization, and is complementary to the cosmological $21 \mathrm{~cm}$ studies. In addition to the mean spectrum, fluctuations in the OI pumping signal may be detectable, provided the background fluctuations on arcminute scales, around $650 \mathrm{GHz}$, can be measured to the nJy level [124]. If the IGM is polluted with metals at high-redshift, then CMB angular fluctuations due to density fluctuations alone (without pumping, just due to the geometrical effect of scattering) could also be detectable for several metal and molecular species [13].

At lower redshift, the details of the later stages of reionization can be probed in more detail, through studying the statistics of Lyman line absorption in the spectra of quasars (e.g. [117, 118] and references therein), gamma-ray burst afterglows, and galaxies (see, e.g., [51] and references therein). In particular, the drop in the cosmic star-formation history near the end-stages of reionization at $z \sim 6-7$ due to photo-heating could be detected in the highredshift extension of the 'Lilly - Madau' diagram [104 109], by directly counting faint galaxies [8]. In practice, the low-mass galaxies susceptible to the reionization suppression are faint and may fall below JWST's detection limit. Whether or not these galaxies will be detected (and in sufficient numbers so that they can reveal the effect of reionization), depends crucially on the feedback effects discussed above, the redshift of reionization, the size of the affected galaxies and their typical star-formation efficiencies, as well as the amount of dust obscuration. Alternatively, by analyzing the Lyman $\alpha$ absorption spectra of SDSS quasars at $z \sim 6$, [29] suggested, from the non-monotonic evolution of the mean IGM opacity, that we may already have detected a drop in the SFR at $z \sim 6$. In order to improve on this current, low signal-to-noise result, deep, high-resolution spectra of bright quasars would be required from beyond the epoch of reionization at $z \gtrsim 6$ (this could be possible with JWST, see [79]). A sup-

pression of low-mass galaxies would also increase the effective clustering of the reionizing sources (since higher-mass halos are more strongly clustered), and increase the fluctuations 
in the Lyman $\alpha$ forest opacity at somewhat lower redshifts. This effect may already have been observed [190], although the modeling details still matter, and the presently measured scatter in opacity could perhaps still be consistent with density fluctuations alone [103].

In $\S$ below, I will return to the issue of feedback, and discuss tracing the cosmic starformation history with distant $\mathrm{SNe}$.

\section{Direct Detections of the First Sources}

The most basic question to ask is whether JWST could directly detect the first stars or black holes. As discussed above, the very first star may have formed in isolation in a $10^{6} \mathrm{M}_{\odot}$ dark matter halo at $z \gtrsim 15$, and it will then be beyond the reach of direct imaging even by JWST. The critical mass for detection with JWST depends on the IMF and star-formation efficiency. At $z=10$, assuming $\sim 10 \%$ of the gas in a halo turns into stars, with a normal Salpeter IMF, a 1nJy broad-band threshold at near-IR wavelengths would allow the detection of a stellar cluster whose mass is a several $\times 10^{6} \mathrm{M}_{\odot}[76$. It will help if the IMF is biased toward more massive, and more luminous stars. If the stars were all metal-free, on the other hand, they would have a lower flux than metal-enriched stars at JWST's wavelengths, due to their high effective temperatures which shifts their flux to (observed) UV wavelengths [176, 22, 148]. The conclusion is that while JWST is very unlikely to detect the first individual stars directly, it will most likely directly measure the luminosity function of faint galaxies, extending down to sufficiently small sizes, corresponding to the halo masses $M=10^{8-10} \mathrm{M}_{\odot}$. This will directly probe the feedback effects discussed above. For example, a clear turn-over in the LF at the luminosity corresponding to the atomic cooling threshold (or lack of it) would be compelling evidence for $\mathrm{H}_{2}$-feedback (or lack of it).

Likewise, one can ask whether JWST can see the first BHs directly? The relevant threshold - i.e. the lowest BH mass - will again depend on the spectrum and luminosity (in terms of, say, the Eddington value) of the BHs. With the average spectrum of quasars at lower redshift [45], and assuming Eddington luminosity, at the 1nJy threshold, JWST could detect a BH whose mass is a several $\times 10^{5} \mathrm{M}_{\odot}$ [77]. If the first BHs are the remnants of massive, metalfree stars, then their initial masses will be below this threshold. Furthermore, it is not clear when such stellar-seed BHs would start shining at a significant fraction of the Eddington limit (since the progenitor star may clear their host halo of gas, as mentioned above). On the other hand, if $\mathrm{H}_{2}$-suppression prevents most Type II halos from forming stars, then the first stars and BHs would appear in large numbers only inside more massive dark halos, with virial temperatures exceeding $T_{\mathrm{vir}} \gtrsim 10^{4} \mathrm{~K}$. How gas cools, condenses, and fragments in such halos is presently not well understood. Using semi-analytical toy models, [128] argued that the initial cooling by atomic $\mathrm{H}$ allows the gas to begin to collapse - even in the face of a significant LW background. If the gas remained at $\sim 10^{4} \mathrm{~K}$, the high Jeans mass $\sim 10^{6} \mathrm{M}_{\odot}$ in these halos would suggest that a supermassive black hole (SMBH) of a similar mass may form at the nucleus [128, 24, 179, 15, 105]. Such BHs could be directly detectable by JWST at $z \sim 10$, provided they shine at $\gtrsim 10 \%$ of their Eddington luminosity.

Predictions for the number counts of high redshift galaxies and quasars at JWST's thresholds at near-infrared wavelengths have been made using simple semi-analytic models [76, 77]. Surface densities as high as several sources per square arcminute are predicted from $z \gtrsim 5$, with most of these sources at $z \gtrsim 10$. These predictions, obtained from the simplest models, represent the DM halo abundance, multiplied with optimistic star/BH formation efficiencies, and corrected for stellar/quasar duty cycles. As such, they could be considered as upper bounds - feedback effects will certainly reduce the counts. If the galaxies occupy a fair fraction $(\sim 5 \%)$ 
of the virial radius of their host halos, then a large fraction $(\gtrsim 50 \%)$ of them can potentially be resolved with JWST's planned angular resolution of $\sim 0.06^{\prime \prime}$ [78, 7].

In addition to broad-band searches, one may look for the earliest light-sources in emission lines. The strongest recombination lines of $\mathrm{H}$ and He from $5<z<20$ will fall in the near-infrared bands of JWST and could be bright enough to be detectable. Specific predictions have been made for the source counts in the $\mathrm{H} \alpha$ emission line [127] and for the three strongest HeII lines that could be powered either by BHs or metal-free stars with a hard spectrum [130, 177]. The key assumption is that most of the ionizing radiation produced by the miniquasars is processed into such recombination lines (rather than escaping into the IGM). Under this optimistic assumption (which is likely violated at least in the smallest minihalos [183]), the lines are detectable for a fiducial $10^{5} M_{\odot}$ miniquasar at $z=10$, or for a "microgalaxy" with a star-formation rate of $1 \mathrm{M}_{\odot} \mathrm{yr}^{-1}$. The simultaneous detection of $\mathrm{H}$ and $\mathrm{He}$ lines would be especially significant. As already argued above, the hardness of the ionizing continuum from the first sources of ultraviolet radiation plays a crucial role in the reionization of the IGM. It would therefore be very interesting to directly measure the ionizing continuum of any $z>6$ source. While this may be feasible at X-ray energies for exceptionally bright sources, the absorption by neutral gas within the source and in the intervening IGM will render the ionizing continuum of high redshift sources inaccessible to direct observation out to $\sim 1 \mathrm{keV}$. The comparison of $\mathrm{H} \alpha$ and HeII line strengths can be used to infer the ratio of HeII to HI ionizing photons, $Q=\dot{N}_{\text {ion }}^{\mathrm{HeII}} / \dot{N}_{\mathrm{ion}}^{\mathrm{HI}}$. A measurement of this ratio would shed light on the nature of the first luminous sources, and, in particular, it could reveal if the source has a soft (stellar) or hard (AGN-like) spectrum. Note that this technique has already been successfully applied to constrain the spectra of sources in several nearby extragalactic HII regions [63]. An alternative method to probe the hardness of the spectrum of the first sources would be to study the thickness of their ionization fronts [151, 197, 99]; this may be possible for bright quasars in Lyman line absorption [99] or in $21 \mathrm{~cm}$ studies [197, 196, 99].

Provided the gas in the high redshift halos is enriched to near-solar levels, several molecular lines may be visible. In fact, $\mathrm{CO}$ has already been detected in the most distant $z=6.41$ quasar [180], and, in fact, spatially resolved [181]. The detectability of CO for high redshift sources in general has been considered by [155] and by [68]. For a star formation rate of $\gtrsim 30 M_{\odot} / \mathrm{yr}$, the $\mathrm{CO}$ lines are detectable at all redshifts $z=5-30$ by the Millimeter Array (the redshift independent sensitivity is due to the increasing CMB temperature with redshift), while the Atacama Large Millimeter Array (ALMA) could reveal fainter CO emission. The detection of these molecular lines will provide valuable information on the stellar content and gas kinematics in the earliest halos.

Finally, the baryons inside high-redshift halos with virial temperatures $T \gtrsim 10^{4} \mathrm{~K}$ need to cool radiatively, in order to condense inside the dark matter potential wells, even before any stars or black holes can form. The release of the gravitational binding energy, over the halo assembly time-scale, can result in a significant and detectable Ly $\alpha$ flux [81 [52]. At the limiting line flux $\approx 10^{-19} \mathrm{erg} \mathrm{s}^{-1} \mathrm{~cm}^{-2}$ asec $^{-2}$ of JWST, several sufficiently massive halos, with velocity dispersions $\sigma \gtrsim 120 \mathrm{~km} \mathrm{~s}^{-1}$, would be visible per $4^{\prime} \times 4^{\prime}$ field. The halos would have characteristic angular sizes of $\approx 10^{\prime \prime}$, would be detectable in a broad-band survey out to $z \approx 6-8$, and would provide a direct probe of galaxies in the process of forming. They may be accompanied by $\mathrm{He}^{+}$Ly $\alpha$ emission at the $\approx 10 \%$ level [191], but remain undetectable at other wavelengths. The main challenge, if such blobs are detected, without any continuum source, will likely be the interpretation - as is the case for the currently detected extended Lyman $\alpha$ sources (there are currently $\sim$ three dozen extended Ly $\alpha$ blobs known, with $1 / 3$ rd of such objects in the largest sample [113] consistent with being powered by cooling radiation). 
Monte Carlo calculations of $\operatorname{Ly} \alpha$ radiative transfer through optically thick, spherically symmetric, collapsing gas clouds were presented in [37, 38], with the aim of identifying a clear diagnostic of gas infall (a similar effort in 3D is being carried out [170]). These represent simplified models of proto-galaxies that are caught in the process of their assembly. Such galaxies can produce Ly $\alpha$ flux over an extended solid angle, either from a spatially extended Ly $\alpha$ emissivity, or from scattering effects, or both. We presented a detailed study of the effect of the gas distribution and kinematics, and of the $\operatorname{Ly} \alpha$ emissivity profile, on the emergent spectrum and surface brightness distribution. The emergent Ly $\alpha$ spectrum is typically doublepeaked and asymmetric. The surface brightness distribution is typically flat, and the detection of a strong wavelength dependence of its slope (with preferential flattening at the red side of the line) would be a robust indication that Ly $\alpha$ photons are being generated (rather than just scattered) in a spatially extended region around the galaxy, as in the case of a cooling flow. An alternative, robust diagnostic for scattering is provided by the polarization of an extended Lyman $\alpha$ source [39]. Spectral polarimetry (in particular, the wavelength-dependence of the polarization) can differentiate between Lya scattering off infalling gas and outflowing gas.

\section{Tracing The Beginning of the Cosmic Star-Formation History With Supernovae}

As argued above, the very first stellar clusters may fall below the direct detection of JWST. A promising way (and probably the only way) to directly observe the first stars individually is through their explosions, either as Supernovae or gamma ray bursts (GRBs). Before we present the expectations for $\mathrm{SNe}$ in detail, we first briefly discuss an alternative, GRB afterglows. In the years leading up to the launch of the Swift satellite 1 it has been increasingly recognized that distant gamma ray bursts (GRBs) offer a unique probe of the high redshift universe. In particular, GRBs are the brightest known electromagnetic phenomena in the universe, and can be detected up to very high redshifts (e.g. [184, 101, 33]), well beyond the redshift $z \approx$ 6.5 of the most distant currently known quasars [50] and galaxies [44]. There is increasing evidence that GRBs are associated with the collapse of short-lived, massive stars, including the association of bursts with star-forming regions (e.g. [55], a contribution of supernova light to the optical afterglow (e.g. [17, 62]), and most directly, association with a supernova [163, 86].

As a result, the redshift distribution of bursts should follow the mean cosmic starformation rate (SFR). Several studies have computed the evolution of the expected GRB rate under this assumption, based on empirical models of the global SFR [174, 175, 184, 101, 33]. Determinations of the cosmic SFR out to redshift $z \sim 5$ (e.g. [26, 60, 64]) have shown that starformation is already significant at the upper end of the measured redshift range, with $\gtrsim 10 \%$ of all stars forming prior to $z=5$, which would result in a significant population of GRBs at these redshifts. Further associating star-formation with the formation rate of non-linear dark matter halos, and using theoretical models based on the Press-Schechter formalism [137], it is possible to extrapolate the SFR and obtain the GRB rates expected at still higher redshifts [23, 31, 120]. These studies have concluded that a significant fraction (exceeding several percent) of GRBs detected at Swift's sensitivity should originate at redshifts as high as $z>10$. The spectra of bright optical/IR afterglows of such distant GRBs can provide information both on the progenitor, and also reveal absorption features by neutral hydrogen in the intergalactic

\footnotetext{
${ }^{1}$ See http://swift.gsfc.nasa.gov
} 
medium (IGM), and can serve as an especially clean probe of the reionization history of the universe [121, 101, 31, 100, 10].

In summary, the main advantage of GRBs is that they are bright and can be seen essentially from arbitrary far away. The main drawback, however, is that they are rare. Even the most optimistic among the above-listed models predict only a handful of detectable GRBs per year from $z>6$ - indeed, in the past two years, only one GRB has been discovered at $z>6$ (GRB050904 at $z=6.3$; [94]).

\subsection{Supernovae From The First Stars}

Although SNe are not as bright as GRB afterglows, they are bright compared to galaxies at the very faint end of the luminosity function, and individual core-collapse SNe would still be visible from beyond $z=10$. Furthermore, since they occur much more frequently than GRBs, and they remain visible for a longer time than GRB afterglows, they will likely offer a better (and possibly only) chance to trace out the cosmic star formation history accurately, with a statistically significant sample extending beyond $z>10$. The above is true even if the bulk of early supernovae are similar to the core-collapse SNe in the local universe (note that Type Ia supernovae will not have time to form at $z \gg 6$ ). In addition, the pair-instability supernovae from massive, metal-free stars are expected to be much brighter than Type II supernovae from normal (metal-enriched) stars [85].

There have been several studies of the expected early supernova rate (SNR), calibrated to the observed metallicity of the Lyman $\alpha$ forest [122] or the observed SFR at lower redshifts [34]. The expected rate of pair-instability SNe from very high- $z$ from the first generation of metal-free stars was studied by [185]. In a recent work [119], we constructed the expected detection rate of high- $z \mathrm{SNe}$ in SNe surveys for JWST. We also quantified the prospects of detecting a drop in the $\mathrm{SN}$ rate due to photo-heating feedback at reionization. Given that $\mathrm{SNe}$ may be our only hope to directly map out the beginning of the cosmic star-formation history, most of the rest of this article is devoted to discussing this possibility in detail.

\subsection{The Global Star Formation and Supernova Rates}

The global SFR density can be obtained and extrapolated by a common approach, based on the dark matter halo formation rate, calibrating the star-formation efficiency to the SFR at redshift $z \lesssim 6$. From this extrapolated SFR density, we can then obtain the intrinsic supernova rate by using the properties of core-collapse SNe in the local universe as a guide.

In particular, we can estimate the global SFR density at redshift $z$ as

$$
\dot{\rho}_{*}(z)=\varepsilon_{*} \frac{\Omega_{b}}{\Omega_{\mathrm{M}}} \int_{M_{\min }(z)}^{\infty} d M \int_{\infty}^{z} d z^{\prime} M \frac{\partial^{2} n\left(>M, z^{\prime}\right)}{\partial M \partial z^{\prime}} P(\tau),
$$

where $\varepsilon_{*}$ is the efficiency (by mass) for the conversion of gas into stars, $\operatorname{MdM}(\partial n(>$ $M, z) / \partial M)$ is the mass density contributed by halos with total (dark matter + baryonic) masses between $M$ and $M+d M, t(z)$ is the age of the universe at redshift $z$, and $P(\tau)$ is the probability per unit time that new stars form in a mass element of age $\tau \equiv t(z)-t\left(z^{\prime}\right)$ (normalized to $\int_{0}^{\infty} d \tau P(\tau)=1$ ). We adopt the fiducial value of $\varepsilon_{*}=0.1$ (see, e.g., [28]). Note that the star formation efficiency in minihalos (i.e. halos with virial temperatures below $10^{4} \mathrm{~K}$ ) that contain pristine metal-free gas could be significantly lower than 0.1 , as suggested by numerical simulations of the first generation of stars [1, 20]. The expected pre-reionization SNe rates would then lie closer to our $T_{\mathrm{vir}} \gtrsim 10^{4} \mathrm{~K}$ curves prior to reionization (see discussion below), making 
the detection of the reionization feature significantly more difficult. However, the efficiency is likely to be very sensitive to even trace amounts of metallicity [21], and conditions for starformation may result in a standard initial mass function (IMF) in gas that has been enriched to metallicities above a fraction $10^{-4}$ of the solar value. Indeed, it is unlikely that metal-free star-formation in minihalos can produce enough ionizing photons to dominate the ionizing background at reionization (e.g. [72, 74, 157]). We assume further that star-formation occurs on an extended time-scale, corresponding to the dynamical time, $t_{\mathrm{dyn}} \sim[G \rho(z)]^{-1 / 2}[30,66$ :

$$
P(\tau)=\frac{\tau}{t_{\text {dyn }}^{2}} \exp \left[-\frac{\tau}{t_{\text {dyn }}}\right],
$$

where $\rho(z) \approx \Delta_{c} \rho_{\text {crit }}(z)$ is the mean mass density interior to collapsed spherical halos (e.g. [9]), and $\Delta_{c}$ is obtained from the fitting formula in [25], with $\Delta_{c}=18 \pi^{2} \approx 178$ in the Einsteinde Sitter model. The minimum mass, $M_{\min }(z)$ in eq. (1), depends on the efficiency with which gas can cool and collapse into a dark matter halo. Prior to reionization and without molecular hydrogen, $M_{\min }(z)$ corresponds to a halo with virial temperature, $T_{\mathrm{vir}} \sim 10^{4} \mathrm{~K}$; with a significant $\mathrm{H}_{2}$ abundance, the threshold decreases to $T_{\text {vir }} \sim 300 \mathrm{~K}$ ([72]; we use the conversion between halo mass and virial temperature as given in [9]). Post reionization, the Jeans mass is raised, so $M_{\min }(z)$ could increase. The degree of self-shielding, the ability of the halo gas to cool, as well as the amount of $\mathrm{H}_{2}$ present in the high-redshift low-mass halos is uncertain [36], and so below we present results for several values of $M_{\min }(z)$, which we will henceforth express in terms of $T_{\mathrm{vir}}(\mathrm{z})$.

Next, from $\dot{\rho}_{*}(z)$ we obtain the intrinsic differential SNR (number of core collapse SNe per unit redshift per year) with

$$
\frac{d \dot{N}}{d z}=\eta_{\mathrm{SN}} \frac{1}{1+z} \frac{d V(z)}{d z} \dot{\rho}_{*}(z),
$$

where the factor $1 /(1+z)$ accounts for time dilation, $d V(z) / d z$ is the comoving volume in our past light cone per unit redshift, and $\eta_{\mathrm{SN}}$ is the number of SNe per solar mass in stars. For a fiducial Salpeter initial mass function (IMF), we obtain $\eta_{\mathrm{SN}} \sim 1 / 180 \mathrm{M}_{\odot}^{-1}$, assuming all stars with masses $9 M_{\odot} \lesssim M \lesssim 40 M_{\odot}$ become core collapse SNe [84]. We neglect the lifetime of these high mass stars in determining our SNR; this is a reasonable assumption as the lifetimes (as well as the spread in the lifetimes) are shorter than a unit redshift interval for redshifts of interest. Note that an alternative extreme shape for the IMF, consisting entirely of 100-200 $M_{\odot}$ stars [1, 20] would yield a similar value for $\eta_{\mathrm{SN}}$.

We present our SFR densities (top panel) and SNRs (bottom panel) in Figure 1 The curves correspond to redshift-independent virial temperature cutoffs of $T_{\mathrm{vir}}=300,10^{4}, 4.5 \times 10^{4}$, and $1.1 \times 10^{5} \mathrm{~K}$ (or circular velocities of $v_{\text {circ }}=3,17,35$, and $55 \mathrm{~km} \mathrm{~s}^{-1}$, respectively), top to bottom, spanning the expected range [173, 36]. Also shown are results from GOODS [64]: the bottom points assume no dust correction and the top points are dust corrected according to [3]; the statistical error bars lie within the points [3]. As there are large uncertainties associated with dust correction, each pair of points (top and bottom) serves to encompass the expected SFR densities.

Our SFRs are consistent with other theoretical predictions (e.g. [158, 8, 23]), as well as other estimates from the Hubble Ultra Deep Field [26] and the FORS Deep Field on the VLT [60], after they incorporate a factor of 5-10 increase in the SFR [3] due to dust obscuration. Furthermore, we note that our SNRs, which at $z \gtrsim 5$ yield $0.3-2$ SNe per square arcminute per year, are in good agreement with the $\sim 1 \mathrm{SN}$ per square arcminute per year estimated by [122] by requiring that high-redshift $\mathrm{SNe}$ produce a mean metallicity of $\sim 0.01 Z_{\odot}$ by 
$z \sim 5$. Note, however, that the rates we obtain are significantly higher (by a factor of $\sim 60$ -2000 at $z \sim 20$ ) than those recently found by [185]. The reason for this large difference is that Wise \& Abel consider star-formation only in minihalos, and they assume a very low starformation efficiency of a single star per minihalo, as may be appropriate for star-formation out of pristine (metal-free) gas in the first generation of minihalos [1, 20]. In contrast, we assume an efficiency of $\varepsilon_{*}=0.1$, which may be more appropriate for star-formation in pre-enriched gas that dominates the SFR just prior to reionization (including star-formation in minihalos).

As mentioned above, by increasing the cosmological Jeans mass, reionization is expected to cause a drop in the SFR (and hence the SNR), with the rates going from the horizontally striped region in Figure 1 at $z>z_{\text {re }}$ to the vertically striped region at $z<z_{\text {re }}$.

The redshift width of this transition is set by a combination of large-scale cosmic variance, radiative transfer, and feedback effects. For the majority of the paper, we use $\Delta z_{\text {re }} \sim 1$ as a rough indicator of the width of the transition we are analyzing. We distinguish between "reionization" and a "reionization feature", and use $\Delta z_{\text {re }}$ as an indicator of the width of the later. Even with an extended reionization history $(\Delta z \sim 10)$, fairly sharp $\left(\Delta z_{\text {re }} \lesssim 3\right)$ features are likely, as discussed in detail in [119].

The other important factor determining the usefulness of the method proposed here is the factor by which the SFR drops during the reionization epoch. The size of this drop is mediated by the effectiveness of self-shielding and gas cooling during photo-heating feedback: i.e. on whether or not the star-formation efficiency is significantly suppressed in those halos that dominate the SFR and SNR immediately preceding the reionization epoch. Given the uncertainties about this feedback discussed above, we will consider a range of possibilities below, parameterized by the modulation in the virial temperature threshold for star-formation during reionization.

\subsection{The Rate of High-Redshift SNe Detectable in a Future Survey}

Given the intrinsic star-formation and SN rates, our next task is to estimate the number of $\mathrm{SNe}$ that could actually be detected in a future search with JWST. In general, the number of SNe per unit redshift, $d N_{\exp } / d z$, that are bright enough to be detectable in an exposure of duration $t_{\text {exp }}$ can be expressed as

$$
\frac{d N_{\exp }}{d z}=\frac{d \dot{N}}{d z} \int_{0}^{\infty} f_{\mathrm{SN}}\left(>t_{\mathrm{obs}}\right) d t_{\mathrm{obs}}
$$

where $(d \dot{N} / d z) d t_{\mathrm{obs}}$ is the number of SNe which occurred between $t_{\mathrm{obs}}$ and $t_{\mathrm{obs}}+d t_{\mathrm{obs}}$ ago (per unit redshift; note that the global mean SNR will evolve only on the Hubble expansion time-scale, and can be considered constant over several years), and $f_{\mathrm{SN}}\left(>t_{\mathrm{obs}}\right)$ is the fraction of SNe which remains visible for at least $t_{\mathrm{obs}}$ in the observed frame. Then the total number of $\mathrm{SNe}$ detected in a survey of duration $t_{\text {surv }}$ is

$$
N_{\text {surv }}=\frac{t_{\text {surv }}}{2 t_{\text {exp }}} \frac{\Delta \Omega_{\mathrm{FOV}}}{4 \pi} N_{\exp }
$$

where $\Delta \Omega_{\mathrm{FOV}}$ is the instrument's field of view, and $t_{\text {surv }} /\left(2 t_{\mathrm{exp}}\right)$ is the number of fields which can be tiled in the survey time, $t_{\text {surv }}$ (we add a factor of $1 / 2$ to allow for a second pair of filters to aid in the photometric redshift determination; note that this provides for imaging in 4 different JWST bands; see discussion below). Note also that equation (5) is somewhat idealized, in that it assumes continuous integration for a year, and e.g., does not account for time required to slew the instrument to observe different fields. In principle, each field has to have repeated 


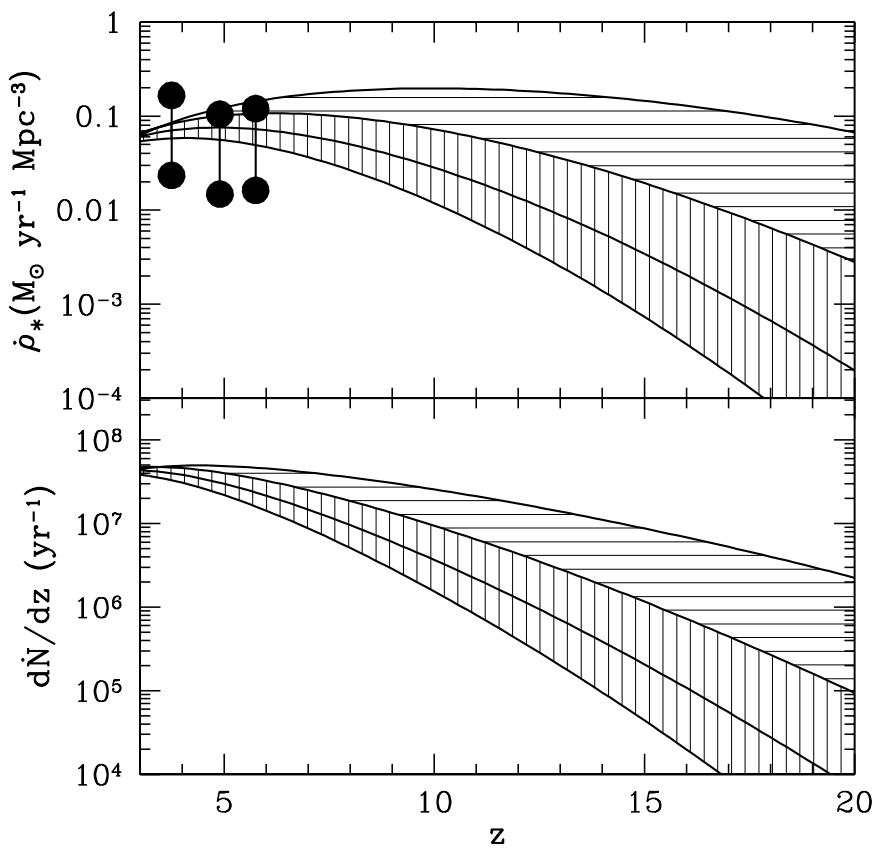

Fig. 1. Upper Panel: SFR densities obtained in a model based on dark matter halo abundances. The curves (top to bottom) correspond to different lower cutoffs on the virial temperatures of star-forming halos, $T_{\text {vir }} \gtrsim 300,10^{4}, 4.5 \times 10^{4}$, and $1.1 \times 10^{5} \mathrm{~K}$ (corresponding to circular velocity thresholds of $v_{\text {circ }} \gtrsim 3,17,35$, and $55 \mathrm{~km} \mathrm{~s}^{-1}$ ). Dots indicate results from GOODS [64]: the lower set of points assume no dust correction, while the upper set of points are dust corrected; statistical 1- $\sigma$ error bars lie within the points. The lines connecting each pair of points span the expected range of SFR densities. Lower Panel: the total global supernova rate accompanying the SFR densities in the top panel (adapted from [119]).

observations (to detect $\mathrm{SNe}$ by their variability), and therefore any dedicated survey should target fields that have already been observed. Furthermore, a dedicated year-long program may not be necessary, because the effect could be detected with relatively few fields (at least under optimistic assumptions; see discussion below), and several fields with repeated imaging (separated by $>1-2$ years) may already be available from other projects; these fields can then be used for the $\mathrm{SN}$ search.

In general, $f_{\mathrm{SN}}\left(>t_{\mathrm{obs}}\right)$ in equation (4), i.e. the fraction of $\mathrm{SNe}$ which remain visible for at least $t_{\mathrm{obs}}$, depends on (i) the properties of the $\mathrm{SN}$, in particular their peak magnitude and lightcurve, and the distribution of these properties among $\mathrm{SNe}$, and (ii) on the properties of the telescope, such as sensitivity, spectral coverage, and field of view. In the next two subsections, we discuss our assumptions and modeling of both of these in turn. 


\section{Empirical Calibration of SN Properties}

At each redshift, we run Monte-Carlo simulations to determine $f_{\mathrm{SN}}\left(>t_{\mathrm{obs}}\right)$ in equation (4). We use the observed properties of local core-collapse $\mathrm{SNe}(\mathrm{CCSNe})$ in estimating $f_{\mathrm{SN}}\left(>t_{\mathrm{obs}}\right)$. For the high redshifts of interest here, we only consider core collapse $\mathrm{SNe}$ of Type II. SNe resulting from the collapse of Chandrasekhar-mass white dwarfs (Type Ia) are expected to be extremely rare at high redshifts $(z \gtrsim 6)$, as the delay between the formation of the progenitor and the SN event ( $\gtrsim 1 \mathrm{Gyr}$; [167]) is longer than the age of the universe at these redshifts. Local core CCSNe come in two important varieties, types IIP and IIL, differentiated by their lightcurve shapes. We ignore the extremely rare additional CCSN types, e.g Type IIn and IIb, which appear to have significant interaction with circumstellar material and constitute less than $10 \%$ of all CCSNe. Type Ib/c, which may or may not also result from core collapse, have luminosities and light-curves that are similar to Type IIL and occur less frequently. While the relative numbers of Type IIP and Type IIL SNe are not known even for nearby SNe, estimates imply that they are approximately equal in frequency [27]. We therefore assume that $50 \%$ of the high-redshift SNe are Type IIP and 50\% are Type IIL.

CCSNe result from the collapse of the degenerate cores of high-mass stars. The luminosity of CCSNe is derived from the initial shock caused by the core-collapse which ionizes material and fuses unstable metal isotopes (see [102] and references therein for a more detailed description of SN lightcurves). In the early stages of the SN, the shock caused by the core collapse breaks out from the surface of the progenitor (typically high mass red giants), resulting in a bright initial peak in the light curve that lasts less than a few days in the rest-frame of the $\mathrm{SN}$. As the shock front cools, the SN dims. However, the SN may then reach a plateau of constant luminosity in the light curve, believed to be caused by a wave of recombining material (ionized in the shock) receding through the envelope. The duration and strength of this plateau depends on the depth and mass of the progenitor envelope, as well as the explosion energy, with those $\mathrm{SNe}$ exhibiting a strong plateau classified as Type IIP. A typical plateau duration is $\lesssim 100$ rest-frame days [135]. In Type IIL SNe, this plateau is nearly non-existent, and the lightcurve smoothly transitions from the rapid decline of the cooling shock to a slower decline where the luminosity is powered by the radioactive decay of metals in the SN nebula. After the plateau, Type IIP SNe also enter this slowly declining 'nebular' phase.

These observationally determined behaviors have been summarized in a useful form as lightcurve templates in [40]. We use these template lightcurves in determining $f_{\mathrm{SN}}\left(>t_{\mathrm{obs}}\right)$, and normalize the lightcurves using Gaussian-distributed peak magnitudes (i.e. log-normally distributed in peak flux) determined by [140] from a large sample of local Type IIP and Type IIL SNe (see Table 2). We perform the Monte-Carlo simulations with both the dust corrected, and dust uncorrected values in [140], since the dust production history of the early universe is poorly understood and is essentially unconstrained empirically.

We use a combined high-resolution HST STIS + ground-based spectrum of the Type IIP supernova SN1999em (the 'November 5th' spectrum of [12]) as the template SN spectrum in order to obtain $K$-corrections (with the [40] lightcurves given in the restframe $B$ filter). This spectrum was obtained within 10 days of maximum light, i.e. during the initial decline of the $\mathrm{SN}$ brightness, and has been dereddened by $\mathrm{A}_{V}=0.3 \mathrm{mag}$ [12] 83]. While the spectrum, and hence the $K$-corrections, of $\mathrm{SNe}$ evolve during the lightcurve, this effect is not strong for the wavelengths of interest [135], especially since the lightcurve template we use is well matched to the wavelengths being probed by the observations we consider below (leading to small $K$-corrections). We have also used this Type IIP SN template spectrum to calculate $K$ corrections for Type IIL SNe. This is necessary due to the lack of restframe UV spectra of Type IIL SNe that can be combined with optical spectra, and justifiable because the $K$-corrections 
are relatively small and the broadband colors of both Type IIP and IIL SNe (a measure of the spectral shape that determines the $K$-corrections) are similar, at least in the optical [135]. Note that we assume that very-high redshift core-collapse $\mathrm{SNe}$ are similar to local $\mathrm{SNe}$ in their spectra, peak luminosities, and temporal evolution. However, these assumption do not significantly impact our conclusions below, as long as (i) the average properties of the $\mathrm{SNe}$ do not change rapidly at high redshift (which could mimic the reionization drop), (ii) they do not become preferentially under-luminous (which would make high- $z$ SNe less detectable, lowering the statistical confidence at which the reionization drop is measured), and (iii) the detection efficiency does not change rapidly with redshift (e.g. due to instrument parameters or spectral lines).

Table 2. Means and standard deviations of the adopted peak absolute magnitudes of core collapse SNe. Values are taken from [140]. Note: an $M_{B}=-17 \mathrm{SN}$ would be detectable out to $z \approx 8.2$ at the flux threshold of $3 \mathrm{nJy}$ in the $4.5 \mu \mathrm{m}$ band with JWST.

\begin{tabular}{ccccc}
\hline \hline \multicolumn{5}{c}{ Corrected for Dust Not Corrected for Dust } \\
\hline SN Type & $\left\langle M_{B}\right\rangle$ & $\sigma$ & $\left\langle M_{B}\right\rangle$ & $\sigma$ \\
\hline \hline IIP & -17.00 & 1.12 & -16.61 & 1.23 \\
IIL & -18.03 & 0.9 & -17.80 & 0.88 \\
\hline \hline
\end{tabular}

In order to use the method outlined above to probe reionization, the $\mathrm{SN}$ redshifts must also be known to an accuracy of $\Delta z \lesssim 1$. SN redshifts can be determined via spectroscopy of either the SN itself, or of the host galaxy. However, as we have already noted, the host galaxies may only be marginally detectable even in imaging, and the SNe may be too faint for anything other than extremely low resolution spectroscopy. We present here only a very brief example of the possibility of obtaining redshifts from the extremely low resolution $(\lambda / \Delta \lambda \sim 5)$ spectra provided by multi-band imaging: a complete investigation of this possibility is warranted, but is beyond the scope of this paper.

To the extent that Type II SNe spectra can be represented as a sequence of blackbodies of different temperatures (e.g. [34]) photometric redshifts will be impossible to obtain without information about the SN epoch, since temperature and redshift would be degenerate. However, local Type IIP SNe show significant deviations from a blackbody in the UV $(\lambda<3500 \AA)$ due to metal-line blanketing in the SN photosphere, providing spectral signatures that could be used as redshift indicators, depending on their strength. In Figure 2 , we show the evolution with redshift of the infrared colors (in bands accessible with JWST; see below) of our template SN spectrum, compared with the color evolution of a blackbody. The figure shows that the template spectrum deviates significantly from a blackbody. If the spectrum of the $\mathrm{SN}$ is always the same as the template spectrum, then there are good prospects for obtaining photometric redshifts for these $\mathrm{SNe}$, at least in the redshift range $z=7-13$. While the figure shows that multiple redshifts may be possible at fixed observed colors, the degenerate solutions would correspond to $z>16 \mathrm{SNe}$; contamination from such high redshift will be mitigated by the fact that these $\mathrm{SNe}$ are likely to be too faint to be detected. Of course, more detailed studies of the $\mathrm{UV}$ behavior of local SNe, especially their variety and spectral evolution, will be necessary to confirm the possible use of photometric redshifts. 


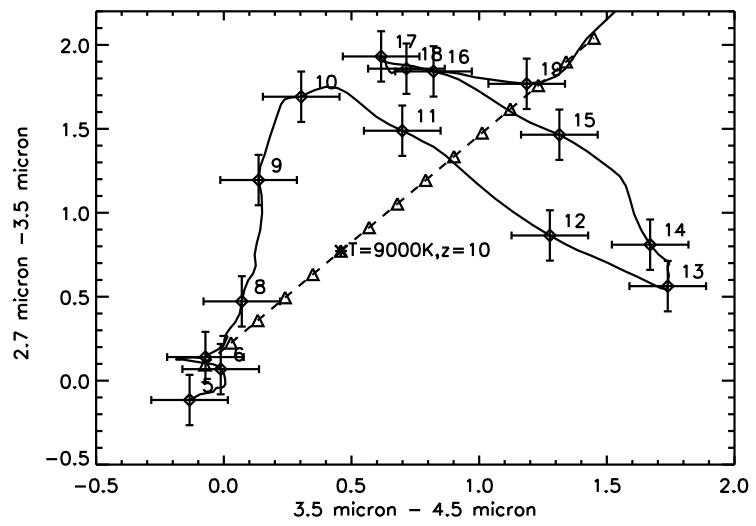

Fig. 2. Infrared colors of a Type II SN as a function of its redshift. Solid curve: The template spectrum of the text, from the Type IIP SN $1999 \mathrm{em}$. Diamonds are placed at intervals of $\Delta z=$ 1 , labeled with the redshift. Error bars of 0.15 mag are shown to give a sense of the photometric errors that may be expected, excluding the many possible systematic effects. Dashed curve: The colors of a blackbody at different redshifts (temperatures) are shown for reference. The asterisk marks the color of a $9000 \mathrm{~K}$ blackbody at $z=10$, triangles are at intervals of $\Delta z=1$; or equivalently, at a constant redshift, but with correspondingly different temperatures (adapted from [119]).

\section{SNe Detectability and Survey Parameters}

As a specific example for the number of detectable SNe in a future sample, we consider observations by JWST. The relevant instrument on JWST is NIRcam 2 a near-infrared imaging detector with a FOV of $2.3^{\prime} \times 4.6^{\prime}$. A field can be observed in two filters simultaneously. NIRcam will have five broadband filters (with resolution $\lambda / \Delta \lambda \sim 5$ ). We model the filter response as tophat functions with central wavelengths of $1.5,2.0,2.7,3.5$, and $4.5 \mu \mathrm{m}$. For concreteness, below we will present results only for the 4.5 and $3.5 \mu \mathrm{m}$ filters, since they are the longest-wavelength JWST bands; however, we allow time for imaging in two other bands, if needed for photometric redshift determinations. The current estimate of the JWST detection threshold at $4.5 \mu \mathrm{m}$ is $\gtrsim 3 \mathrm{nJy}$ for a $10 \sigma$ detection and an exposure time of $10^{5} \mathrm{~s}$. The 3.5 $\mu \mathrm{m}$ band is more sensitive, with a detection threshold of $\gtrsim 1 \mathrm{nJy}$ for a $10 \sigma$ detection and an exposure time of $10^{5} \mathrm{~s}$.

We show our results for $f_{\mathrm{SN}}\left(>t_{\mathrm{obs}}\right)$ in Figure 3 The solid curves correspond to $z=7$, the dashed curves correspond to $z=10$, and the dotted curves correspond to $z=13$. In each panel, the top set of curves assumes a flux threshold of $3 \mathrm{nJy}$ (or an exposure time of $t_{\mathrm{exp}}=10^{5}$ seconds in the $4.5 \mu \mathrm{m}$ band), and the bottom set assumes $9.5 \mathrm{nJy}\left(t_{\exp }=10^{4}\right.$ seconds in the $4.5 \mu \mathrm{m}$ band, background dominated). The top panel further assumes no dust extinction, while in the bottom panel, we adopt the same dust extinction as in the low redshift sample [140]. Understandably, the distributions get wider as redshift increases (due to time dilation), but the total visible fraction, $f_{\mathrm{SN}}(>0)$, gets smaller (due to the increase in luminosity distance).

\footnotetext{
${ }^{2}$ See http://ircamera.as.arizona.edu/nircam for further details.
} 


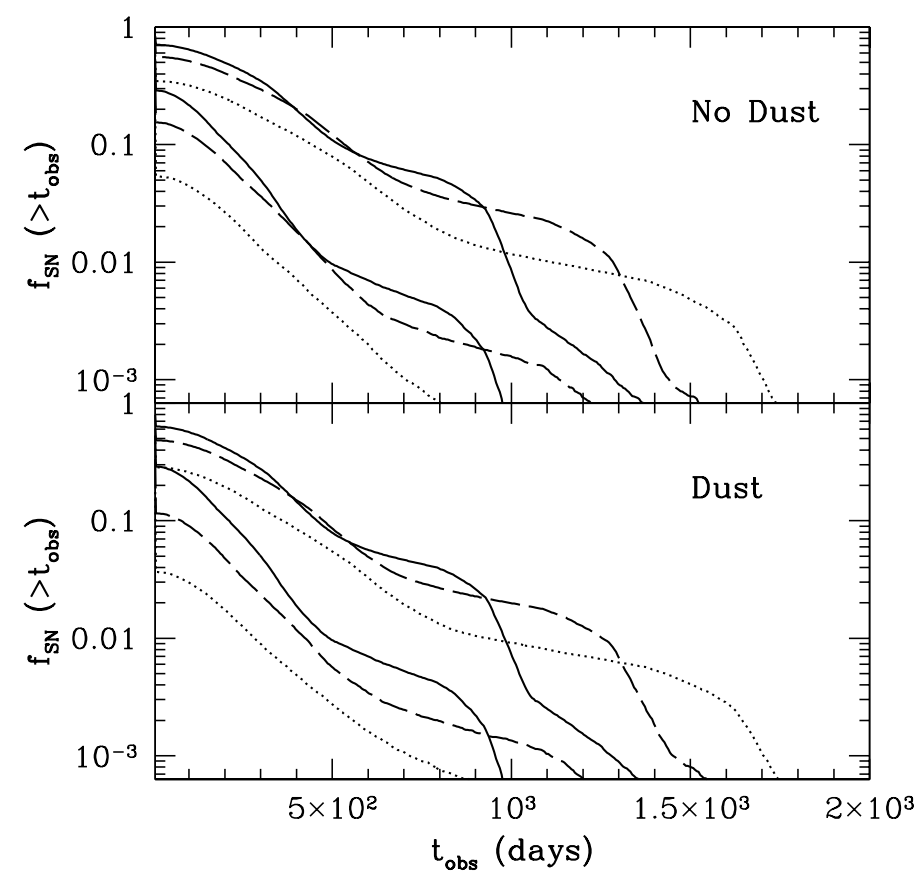

Fig. 3. The fraction of $\mathrm{SNe}$ which remain visible for an observed duration of $t_{\mathrm{obs}}$ or longer. The curves correspond to SN redshifts of $z=7$ (solid curve), $z=10$ (dashed curve), and $z=13$ (dotted curve). In each panel, the top set of curves assumes a flux threshold of $3 \mathrm{nJy}$ (exposure time of $t_{\exp }=10^{5} \mathrm{~s}$ with the $4.5 \mu \mathrm{m} J W S T$ band), and the bottom set assumes $9.5 \mathrm{nJy}$ $\left(t_{\exp }=10^{4} \mathrm{~s}\right)$. The top panel assumes no dust extinction, while the bottom panel assumes the same dust extinction as observed within the low-redshift sample [140] (adapted from [119]).

The double bump feature in some of the curves corresponds to the plateau of Type IIP SNe lightcurves discussed above.

As seen in Figure 3 most of the supernovae that are bright enough to be visible at all, will remain visible for up to $\sim 1-2$ years. Hence, in order to catch the most SNe, it will be necessary to have repeat observations of the SN survey fields a few years apart, to insure that most of the observed SNe will be identified as new sources or sources that have disappeared. A time between observations that is comparable to or larger than the SN duration will be the optimal strategy for detecting the most $\mathrm{SNe}[126]$. Note that we ignore the time required to collect reference images, since observations conducted for other programs will likely provide a sufficient set of such reference images. However, as shown in equation (5), we allow time for the field to be imaged in four different bands, to aid in photometric redshift determination.

To be more explicit, we find that in order to obtain the largest number of high-redshift $\mathrm{SNe}$, in general it is a more efficient use of JWST's time to 'tile' multiple fields rather than 'stare' for extended periods of time $\left(\gtrsim 10^{4} \mathrm{~s}\right)$ at the same field [126]. This is because of strong time dilation at these redshifts. As can be seen from Figure 3 , most detectable $\mathrm{SNe}$ will remain above the detection threshold for several months, even assuming $10^{4} \mathrm{~s}$ integration times. Also evident from Figure 3 is that the increase in the total visible fraction of SNe going 
from $t_{\exp }=10^{4} \mathrm{~s}$ to $t_{\exp }=10^{5} \mathrm{~s}$, is less than the factor of 10 increase in exposure time. As a result, a fiducial 1-yr JWST survey would therefore detect more SNe using $t_{\exp }=10^{4} \mathrm{~s}$ than using $t_{\exp }=10^{5} \mathrm{~s}$ (see Figures 4 and 5). Understandably, this conclusion does not hold for very high-redshifts, $z \gtrsim 14$, where $\mathrm{SNe}$ are extremely faint, and require very long exposure times to be detectable. However, even with such long exposure times, very few SNe will be detectable at these large redshifts, rendering the use of longer exposure times unnecessary.

\section{SNe Detection Rates}

The number of SNe that could be detectable in putative future surveys are shown in Figures 4 and 5 The curves correspond to the same virial temperature cutoffs for star-forming halos as in Figure 1 Solid lines assume no dust obscuration; dashed lines include a correction for dust obscuration as discussed above. Figure 4 shows results assuming flux density thresholds of $9.5 \mathrm{nJy}$ (or $t_{\exp }=10^{4} \mathrm{~s}$ with the $4.5 \mu \mathrm{m} J W S T$ filter) (top panel) and $3 \mathrm{nJy}\left(t_{\exp }=10^{5} \mathrm{~s}\right.$ ) (bottom panel). Figure 5 shows results with the $3.5 \mu \mathrm{m}$ filter assuming equivalent exposure times: flux density thresholds of $3.2 \mathrm{nJy}\left(t_{\exp }=10^{4} \mathrm{~s}\right.$ with the $3.5 \mu \mathrm{m} J W S T$ filter) (top panel) and $1 \mathrm{nJy}\left(t_{\exp }=10^{5} \mathrm{~s}\right)$ (bottom panel). The right vertical axis displays the number of $\mathrm{SNe}$ per unit redshift per FOV $\left(2.3^{\prime} \times 4.6^{\prime}\right)$; the left vertical axis shows the number of SNe per unit redshift in a fiducial 1-year survey. As mentioned above, reionization should be marked by a transition from the region bounded by the top two solid curves to the region enclosed by the bottom three solid curves (or the analog with the dashed curves if dust is present at the time of reionization).

We note that our expected rates are somewhat higher than those in [34], a previous study which included SNe lightcurves and spectra in the analysis. For example, we find $4-24 \mathrm{SNe}$ per field at $z \gtrsim 5$ in the $4.5 \mu \mathrm{m}$ filter with $t_{\exp }=10^{5} \mathrm{~s}$, compared to $\sim 0.7 \mathrm{SNe}$ per field at $z \gtrsim 5$ obtained by [34] (after updating their JWST specifications to the current version). However, they use SFRs extrapolated from the low-redshift data available at the time, which are not a good fit to recent high- $z$ SFR estimates [64, 60, 26], and are lower than our $z \gtrsim 5$ SFRs by a factor of $6-403$ Taking this factor into account, their procedure yields $4-27$ SNe per field at $z \gtrsim 5$, which is in excellent agreement with our estimate of $4-24$ SNe per field at $z \gtrsim 5$.

\section{Pop-III SNe}

As discussed above, high-redshift SNe whose progenitor stars are formed from metal-free gas within minihalos could be intrinsically very different from the low-redshift $\mathrm{SNe}$, due to differences in the progenitor environments (e.g. very low metallicities; [1, 20]). If such differences could be identified and detected, then these "pop-III" SNe could provide valuable information about primordial stars and their environments. Indeed, [185] studied the redshift-distribution of such primordial SNe, but only briefly addressed the issue of their detectability. In order to directly assess the number of such SNe among the hypothetical SNe samples we obtained here, in Figure 6 we plot the fraction of SNe whose progenitor stars are located in minihalos. The solid curve assumes our fiducial model with a Salpeter IMF and $\varepsilon_{* \text { minihalo }}=0.1$; the dotted curve assumes that each minihalo produces only a single star, and hence a single SN, over a dynamical time (assuming that strong feedback from this star disrupts any future star

\footnotetext{
${ }^{3}$ The possibility that the SFR in a "Lilly-Madau diagram" remains flat, or even increases, at redshifts $z \gtrsim 5$, owing to star-formation in early, low-mass halos, is also expected theoretically (see, e.g., Fig.1 in [23] and associated discussion).
} 


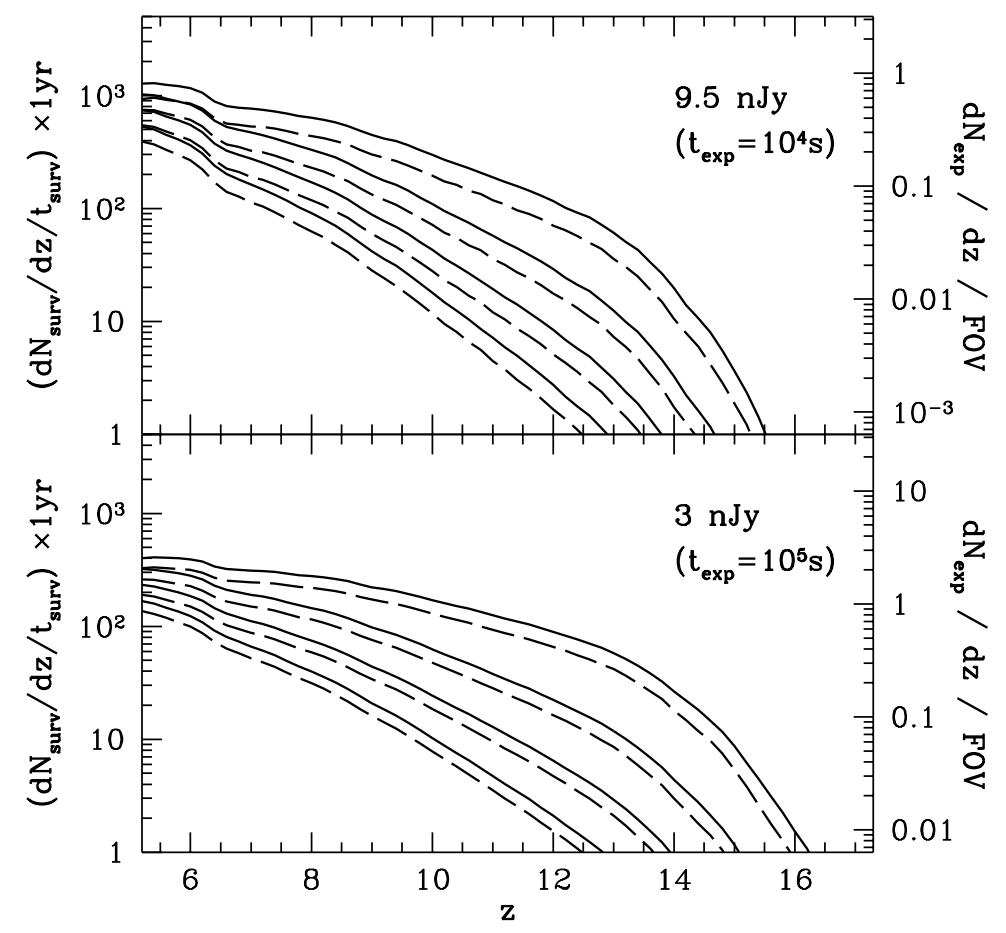

Fig. 4. The number of high-redshift SNe detectable with the $4.5 \mu \mathrm{m} J W S T$ filter. The curves correspond to the same virial temperature cutoffs as in Figure 1 Solid curves assume no dust obscuration; dashed curves adopt dust obscuration in the same amount as observed in the low redshift $\mathrm{SNe}$ sample. The figure shows results assuming flux density thresholds of $9.5 \mathrm{nJy}$ (or $t_{\exp }=10^{4} \mathrm{~s}$ with JWST) (top panel) and $3 \mathrm{nJy}$ (or $t_{\exp }=10^{5} \mathrm{~s}$ with JWST) (bottom panel). The right vertical axis displays the number of SNe per unit redshift per field; the left vertical axis shows the number of SNe per unit redshift found in $t_{\text {surv }} /\left(2 t_{\exp }\right)$ such fields (i.e. the differential version of eq. (5) with $t_{\text {surv }}=1 \mathrm{yr}$ ). Reionization should be marked by a transition from the region bounded by the top two solid curves to the region enclosed by the bottom three solid curves (or the analog with the dashed curves if dust is present at the time of reionization). Adapted from [119].

formation; as in [185]). The figure shows that with an unevolving star formation efficiency, progenitor stars in minihalos would account for $\gtrsim$ half of the SNe at $z \gtrsim 9$. On the other hand, in the extreme case of a single SNe per minihalo, progenitor stars in minihalos would account for less than $\sim 1 \%$ of the $z \sim 10 \mathrm{SNe}$ (although at the earliest epochs, $z \gtrsim 22$, they would still constitute $\gtrsim$ half of all SNe). Given the overall detection rate of several hundred $z \gtrsim 10 \mathrm{SNe}$ with a 1 year JWST survey we have found above, even in this extreme case, Figure 6 implies that several of these SNe could be caused by pop-III stars. 


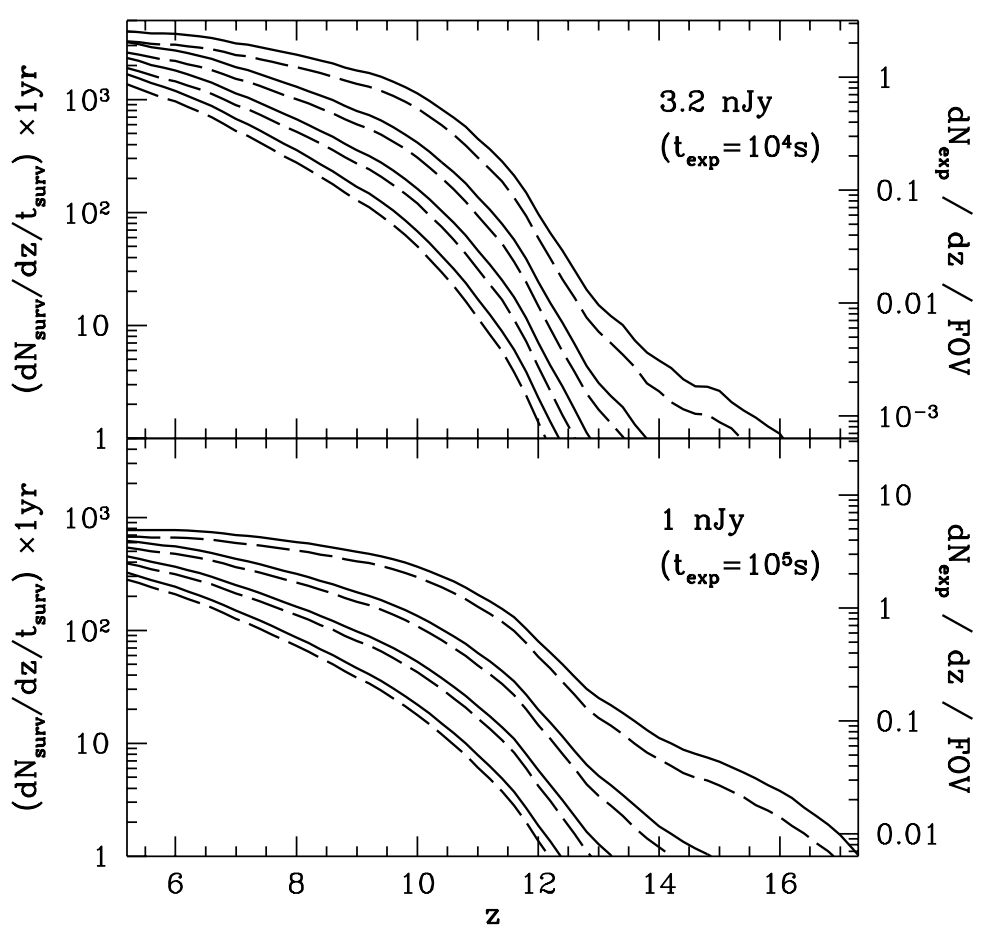

Fig. 5. Same as Figure 4, but with the $3.5 \mu \mathrm{m}$ filter, instead of the $4.5 \mu \mathrm{m}$ one. Note that we present results for comparable exposure times, hence the sensitivity thresholds are three times lower than in Figure 4, due to the disparate sensitivities of the $3.5 \mu \mathrm{m}$ and $4.5 \mu \mathrm{m}$ filters (adapted from [119]).

\subsection{Detecting Features due to Feedback}

In summary, we find that $4-24 \mathrm{SNe}$ may be detectable from $z \gtrsim 5$ at the sensitivity of 3 nJy (requiring $10^{5} \mathrm{~s}$ exposures in a $4.5 \mu \mathrm{m}$ band) in each $\sim 10 \operatorname{arcmin}^{2} J W S T$ field. In a hypothetical one year survey, we expect to detect up to thousands of SNe per unit redshift at $z \sim 6$. These rates are high, and, if reionization produces a fairly sharp features in the reionization history (with a drop in the SFR by a factor of a few, spread at most over $\Delta z_{\text {re }} \sim$ $1-3$ ), we have also shown [119] that the number of SNe is sufficient to detect the feature out to $z \sim 13$, as well as set constraints on the photo-ionization heating feedback on lowmass halos at the reionization epoch. Specifically, for a wide range of scenarios at $z_{\text {re }} \lesssim 13$, the drop in the SNR due to reionization can be detected at $\mathrm{S} / \mathrm{N} \gtrsim 3$ with only tens of deep $J W S T$ exposures. These results therefore suggest that future searches for high $-z \mathrm{SNe}$ could be a valuable new tool, complementing other techniques, to study the process of reionization, as well as the feedback mechanism that regulates it. 


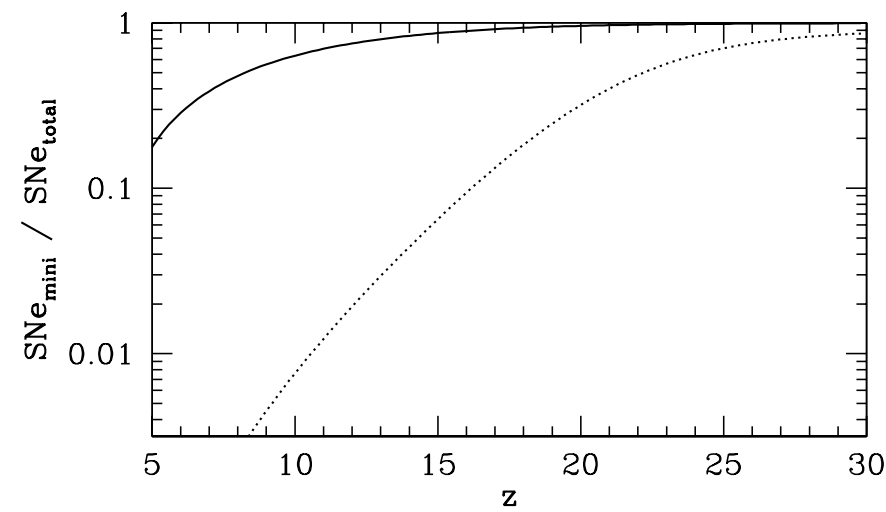

Fig. 6. The fraction of SNe whose progenitor stars are located in minihalos (with virial temperatures of $T_{\mathrm{vir}}<10^{4} \mathrm{~K}$ ). These $\mathrm{SNe}$ may be pair instability $\mathrm{SNe}$, which would be much brighter than the normal core-collapse $\mathrm{SNe}$ used in the computation of SN rates in the previous figures. The solid curve assumes our fiducial model with a Salpeter IMF and $\varepsilon_{* \text { minihalo }}=0.1$; the dotted curve assumes that each minihalo produces only a single star (and therefore a single SN) over a dynamical time (adapted from [119]).

\section{The Dark Ages as a Probe of The Small Scale Power Spectrum}

Since, as discussed above, GRBs are a rare tracer of the cosmic SFR, a mere presence of a GRB at, say, $z>10$ will indicate that non-linear structures exist already at this redshift: the stars that give birth to the GRBs must form out of gas that collected inside dense dark matter potential wells. Structure formation in a cold dark matter (CDM) dominated universe is "bottom-up", with low-mass halos condensing first. In the current "best-fit" cosmology, with densities in CDM and "dark energy" of $\left(\Omega_{\mathrm{M}}, \Omega_{\Lambda}\right) \approx(0.3,0.7)$ that has emerged from WMAP and other recent experiments, DM halos with the masses of globular clusters, $10^{5-6} M_{\odot}$, condense from $\sim 3 \sigma$ peaks of the initial primordial density field as early as $z \sim 25$. It is natural to identify these condensations as the sites where the first astrophysical objects, including the first massive stars, were born. As a result, one expects to possibly find GRBs out to this limiting redshift, but not beyond.

With a scale-invariant initial fluctuation power spectrum, the CDM theory has been remarkably successful, and matched many observed properties of large-scale structures in the universe, and of the cosmic microwave background radiation. However, the power spectrum on scales corresponding to masses of $M \lesssim 10^{9} \mathrm{M}_{\odot}$ remains poorly tested. Some observations have suggested that the standard model predicts too much power on small scales: it predicts steep cusps at the centers of dark matter halos, whereas the rotation curves of dwarf galaxies suggest a flat core; it also predicts more small satellites than appear to be present in the Local Group (reviewed by, e.g. [149]). Although astrophysical explanations of these observations are possible, several proposals to solve the problem have been put forward that involve the properties of dark matter. These include self-interacting dark matter [161], adding a repulsive interaction to gravity [69, 136], the quantum-mechanical wave properties of ultra-light dark matter particles [87], and a resurrection of warm dark matter (WDM) models [18]. 
By design, a common feature of models that attempt to solve the apparent small-scale problems of CDM is the reduction of fluctuation power on small scales. The loss of smallscale power modifies structure formation most severely at the highest redshifts, where the number of self-gravitating objects is reduced. In each model, there exists a redshift beyond which the number of GRBs (or any other object) is exponentially suppressed; a detection of a GRB beyond this redshift can be used to rule out such models. As an example, ref. [6] showed that in the case of WDM models, invoking a WDM particle mass of $\sim 1 \mathrm{keV}$ (the mass needed to solve the problems mentioned above), it becomes difficult to account for the reionization of the universe by redshift $z \sim 6$, due to the paucity of ionizing sources beyond $z=6$.

GRBs, if discovered in sufficient numbers at $z>6$, have the potential to improve this constraints significantly, since increasingly higher redshifts probe increasingly smaller scales. The constraints, in particular, that the detection of distant GRBs would place on structure formation models with reduced small-scale power was quantified in [120]. In this work, we computed the number of GRBs that could be detectable by the Swift satellite at high redshifts $(z \gtrsim 6)$, assuming that the GRBs trace the cosmic star formation history, which itself traces the formation of non-linear structures. Simple models of the intrinsic luminosity function of the bursts were calibrated to the number and flux distribution of GRBs observed by the Burst And Transient Source Experiment (BATSE). Under these assumptions, the discovery of high $-z$ GRBs would imply strong constraints on models with reduced small-scale power. For example, a single GRB at $z \gtrsim 10$, or, alternatively, 10 GRBs at $z \gtrsim 5$, discovered within a two-year period, would rule out an exponential suppression of the power spectrum on scales below $R_{c}=0.09 \mathrm{Mpc}$ (exemplified by warm dark matter models with a particle mass of $m_{x}=2$ $\mathrm{keV}$ ). Models with a less sharp suppression of small-scale power, such as those with a red tilt or a running scalar index, $n_{s}$, are more difficult to constrain, because they are more degenerate with an increase in the power spectrum normalization, $\sigma_{8}$, and with models in which starformation is allowed in low-mass minihalos. We find that a tilt of $\delta n_{s} \approx 0.1$ is difficult to detect; however, an observed rate of $1 \mathrm{GRB} / \mathrm{yr}$ at $z \gtrsim 12$ would yield an upper limit on the running of the spectral index, $\alpha \equiv d n_{s} / d \ln k>-0.05$.

Acknowledgement. This article draws on joint work with numerous colleagues over the past several years. I would like to thank all my collaborators, but especially Andrei Mesinger, Ben Johnson, Greg Bryan, Mark Dijkstra, and Roban Kramer, whose recent works were particularly emphasized here. The work described in this review was supported by NASA, the NSF, and by the the Polányi Program of the Hungarian National Office for Research and Technology (NKTH).

\section{References}

1. Abel, T., Bryan, G. L., \& Norman, M. L. 2002, Science, 295, 93

2. Abel, T., \& Haiman, Z. 2000, in Molecular Hydrogen in Astrophysics, eds. F. Combes and G. P. des Forets, Cambridge University Press, pp. 237-246

3. Adelberger, K. L., \& Steidel, C. C. 2000, ApJ, 544, 218

4. Ahn, K., \& Shapiro, P. R. 2007, MNRAS, 375, 881

5. Bahcall, N.A., Ostriker, J. P., Perlmutter, S., \& Steinhardt, P. J. 1999, Science, 284, 1481

6. Barkana, R., Haiman, Z., \& Ostriker, J. P. 2001, 539, 20

7. Barkana, R., \& Loeb, A. 2000a, ApJ, 531, 613

8. Barkana, R., \& Loeb, A. 2000b, ApJ, 539, 20 
9. - 2001, Phys. Reports, 349, 125

10. - 2004a, ApJ, 601, 64

11. - 2004b, ApJ, 609, 474

12. Baron, E. et al. 2000, ApJ, 545, 444

13. Basu, K., Hernandez-Monteagudo, C., \& Sunyaev, R. 2004, A\&A, 416, 447

14. Becker, R. H., et al. 2001, AJ, 122, 2850

15. Begelman, M., Volonteri, M., \& Rees, M. J. 2006, MNRAS, 370, 289

16. Bennett, C. L., et al. 2003, ApJ, 583, 1

17. Bloom, J. S., et al. 1999, Nature, 401, 453

18. Bode, P., Ostriker, J. P., \& Turok, N. 2001, ApJ, 556, 903

19. Bouwens, R. J., Illingworth, G. D., Blakeslee, J. P., Franx, M. 2006, ApJ, 653, 53

20. Bromm, V., Coppi, P. S., \& Larson, R. B. 2002, ApJ, 564, 23

21. Bromm, V., Ferrara, A., Coppi, P. S., \& Larson, R. B. 2001, MNRAS, 328, 969

22. Bromm, V., Kudritzki, R. P., \& Loeb, A. 2001, ApJ, 552, 464

23. Bromm, V., \& Loeb, A. 2002, ApJ, 575, 111

24. Bromm, V., \& Loeb, A. 2003, ApJ, 596, 34

25. Bryan, G. L., \& Norman, M. L. 1998, ApJ, 495, 80

26. Bunker, A. J., Stanway, E. R., Ellis, R. S., \& McMahon, R. G. 2004, MNRAS, 355, 374

27. Cappellaro, E., Turatto, M., Tsvetkov, D. Y., Bartunov, O. S., Pollas, C., Evans, R., \& Hamuy, M. 1997, A\&A, 322, 431

28. Cen, R. 2003, ApJ, 591, 12

29. Cen, R., \& McDonald, P. 2002, ApJ, 570, 457

30. Cen, R., \& Ostriker, J. P. 1992, ApJ1, 399, L113

31. Choudhury, T. R., \& Srianand, R. 2002, MNRAS, 336, L27

32. Ciardi, B., Ferrara, A., \& Abel, T. 2000, ApJ, 533, 594

33. Ciardi, B. \& Loeb, A. 2000, ApJ, 540, 687

34. Dahlén, T., \& Fransson, C. 1999, A\&A, 350, 349

35. Dickinson, M., et al. 2004, ApJ, 600, L49

36. Dijkstra, M., Haiman, Z., Rees, M. J., \& Weinberg, D. H. 2004, ApJ, 601, 666

37. Dijkstra, M., Haiman, Z., \& Spaans, M. 2006a, ApJ, 649, 14

38. Dijkstra, M., Haiman, Z., \& Spaans, M. 2006b, ApJ, 649, 37

39. Dijkstra, M., \& Loeb, A 2007, ApJ, submitted, arxiv preprint astro-ph:0711.2312

40. Doggett, J. B., \& Branch, D. 1985, AJ, 90, 2303

41. Dove, J. B., Shull, J. M., \& Ferrara, A. 2000, ApJ, 531, 846

42. Dunkley, J., et al. 2008, ApJ, submitted, arXiv.org:0803.0586

43. Efstathiou, G. 1992, MNRAS, 256, 43P

44. Ellis, R. S. 2008, in 'First Light in Universe', Saas-Fee Advanced Courses, No. 36, Swiss Soc. Astrophys. Astron., p. 259

45. Elvis, M., Wilkes, B. J., McDowell, J. C., Green, R. F., Bechtold, J., Willner, S. P., Oey, M. S., Polomski, E., \& Cutri, R. 1994, ApJS, 95, 1

46. Fan, X., et al. 2001, AJ, 122, 2833

47. Fan, X., et al. 2003, AJ, 125, 1649

48. Fan, X. et al. 2004, AJ, 128, 515

49. Fan, X., et al. 2006, AJ, 131, 1203

50. Fan, X. 2006, New Astronomy Reviews, 50, 665

51. Fan, X., Carilli, C. L.., \& Keating, B. 2006, ARA\&A, 44, 415

52. Fardal, M. A., Katz, N., Gardner, J. P., Hernquist, L., Weinberg, D. H., \& Davé, R. 2001, ApJ, 562, 605

53. Ferrara, A. 1998, ApJ, 499, L17 
54. Ferrara, A. 2007, in "Chemodynamics: From First Stars to Local Galaxies", Eds. E. Emsellem, H. Wozniak, G. Massacrier, J.-F. Gonzalez, J. Devriendt and N. Champavert, EAS Publications Series, 24, pp. 229-243

55. Fruchter, A. S., et al. 1999, ApJ, 519, L13

56. Fukugita, M., Hogan, C. J., \& Peebles, P. J. E. 1998, ApJ, 503, 518

57. Furlanetto, S. R., Oh, S.-P., \& Briggs, F. 2006, Physics Reports, 433, 181

58. Furlanetto, S., \& Loeb, 2005, ApJ, 634, 1

59. Furlanetto, S. R., Zaldarriaga, M., \& Hernquist, L. 2004, ApJ, 613, 1

60. Gabasch, A. et al. 2004, ApJ1, 616, L83

61. Gao, L., \& Theuns, T. 2007, Science, 317, 1527

62. Garnavich, P., et al. 2003, ApJ, 582, 2003

63. Garnett, D. R., Kennicutt, R. C., Chu, Y.-H., \& Skillman, E. D. 1991, ApJ, 373, 458

64. Giavalisco, M. et al. 2004, ApJl, 600, L103

65. Glover, S. C. O., \& Brandt, P. W. J. L. 2003, MNRAS, 304, 210

66. Gnedin, N. Y. 1996, ApJ, 456, 1

67. - 2000, ApJ, 542, 535

68. Gnedin, N. Y., Silk, J., \& Spaans, M. 2001, MNRAS,

69. Goodman, J. 2000, New Astronomy, 5, 103

70. Greif, T. H., \& Bromm, V. 2006,MNRAS, 373, 128

71. Haiman, Z. 2004, in Carnegie Observatories Astrophysics Series, Vol. 1: Coevolution of Black Holes and Galaxies, ed. L. C. Ho (Cambridge: Cambridge Univ. Press), pp. 67-87

72. Haiman, Z., Abel, T., \& Rees, M. J. 2000, ApJ, 534, 11

73. Haiman, Z., \& Bryan, G. L. 2006, ApJ, 650, 7

74. Haiman, Z., \& Holder, G. P. 2003, ApJ, 595, 1

75. Haiman, Z., \& Knox, L. 1999, in "Microwave Foregrounds", ASP Conference Series \#181, eds. A. de Oliveira-Costa and M. Tegmark, p.227

76. Haiman, Z., \& Loeb, A. 1997, ApJ, 483, 21

77. Haiman, Z., \& Loeb, A. 1998, ApJ, 503, 505

78. Haiman, Z., \& Loeb, A. 1998a, in "Science with the NGST", Eds. E.P. Smith \& A. Koratkar. (San Francisco: ASP Conference Series), 133, p251

79. Haiman, Z., \& Loeb, A. 1999, ApJ, 519, 479

80. Haiman, Z., Rees, M. J., \& Loeb, A. 1997, ApJ, 476, 458

81. Haiman, Z., Spaans, M., \& Quataert, E. 2000, ApJL, 537, L5

82. Haiman, Z., Thoul, A. A., \& Loeb, A. 1996, ApJ, 464, 523

83. Hamuy, M. et al. 2001, ApJ, 558, 615

84. Heger, A., Fryer, C. L., Woosley, S. E., Langer, N., \& Hartmann, D. H. 2003, ApJ, 591, 288

85. Heger, A., \& Woosley, S. E. 2002, ApJ, 567, 532

86. Hjörth, J., et al. 2003, Nature, 423, 847

87. Hu, W., Barkana, R., \& Gruzinov, A. 2000, PRL, 85, 1158

88. Iliev, I. T., et al. 2006, MNRAS, 371, 1057

89. Iliev, I. T., Mellema, G., Shapiro, P. R.; Pen, U.-L. 2007, MNRAS, 376, 534

90. Iliev, I. T., Pen, U.-L., Bond, J. R., Mellema, G., \& Shapiro, P. R. 2007, ApJ, 660, 933

91. Johnson, J. L., Greif, T. H., \& Bromm, V. 2007, ApJ, 665, 85

92. Kaplinghat, M., Chu, M., Haiman, Z., Holder, G. P., Knox, L., \& Skordis, C. 2003, ApJ, 2003, 583, 25

93. Kashikawa, N., et al. 2006, ApJ, 648, 7

94. Kawai, N. et al. 2006, Nature, 440, 184

95. Kitayama, T., \& Ikeuchi, S. 2000, ApJ, 529, 615 
96. Komatsu, E., et al. 2008, ApJS, submitted, arXiv.org:0803.0547

97. Kuhlen, M., \& Madau, P. 2005, MNRAS, 363, 1069

98. Kramer, R., Haiman. Z., \& Oh, S. P. 2006, ApJ, 649, 570

99. Kramer, R., \& Haiman. Z., MNRAS, submitted, arxiv preprint astro-ph:0712.3548

100. Lamb, D., \& Haiman, Z. 2003, in the proceedings of the 3rd Rome Workshop on Gamma-Ray Bursts in the Afterglow Era, held in Rome, September 2002, eds. L. Piro, F. Frontera, N. Masetti and M. Feroci, ASP Conf. Series, astro-ph/0312502

101. Lamb, D. Q., \& Reichart, D. E. 2000, ApJ, 536, L1

102. Leibundgut, B., \& Suntzeff, N. B. 2003, LNP Vol. 598: Supernovae and Gamma-Ray Bursters, 598, 77

103. Lidz, A., Oh, S. P. \& Furlanetto, S. R. 2006, ApJL, 639, 47

104. Lilly, S. J., Le Fevre, O., Hammer, F., \& Crampton, D. 1996, ApJ1, 460, L1

105. Lodato, G., \& Natarajan, P. 2006, MNRAS, 371, 1813

106. Machacek, M. E., Bryan, G. L., Abel, T. 2001, ApJ, 548, 509

107. Machacek, M. E., Bryan, G. L., Abel, T. 2003, MNRAS, 338, 273

108. MacLow, M.-M. \& Ferrara, A. 1999, ApJ, 513, 142

109. Madau, P., Ferguson, H. C., Dickinson, M. E., Giavalisco, M., Steidel, C. C., \& Fruchter, A. 1996, MNRAS, 283, 1388

110. Madau, P., Rees, M. J., Volonteri, M., Haardt, F. \& Oh, S. P. 2004, ApJ, 604, 484

111. Malhotra, S., \& Rhoads, J. E. 2004, ApJ, 617, L5

112. Malhotra, S., et al. 2005, ApJ, 626, 666

113. Matsuda, Y., Yamada, T., Hayashino, T., Tamura, H., Yamauchi, R., Ajiki, M., Fujita, S. S., Murayama, T., Nagao, T., Ohta, K., Okamura, S., Ouchi, M., Shimasaku, K., Shioya, Y., \& Taniguchi, Y. 2004, AJ, 128, 569

114. McQuinn, M., Furlanetto, S. R.; Hernquist, L., Zahn, O., \& Zaldarriaga, M. 2005, ApJ, 630, 643

115. Salvaterra, R., Ciardi, B., Ferrara, A., \& Baccigalupi, C. 2005, MNRAS, 360, 1063

116. Mesinger, A., Bryan, G. L. , \& Haiman, Z. 2006, ApJ, 648, 835

117. Mesinger, A., \& Haiman, Z. 2004, ApJL, 611, L69

118. Mesinger, A., \& Haiman, Z. 2007, ApJ, 660, 923

119. Mesinger, A., Johnson, B., \& Haiman, Z. 2006, ApJ, 637, 80

120. Mesinger, A., Perna, R., \& Haiman, Z. 2005, ApJ, 623, 1

121. Miralda-Escudé, J. 1998, ApJ, 501, 15

122. Miralda-Escudé, J., \& Rees, M. J. 1997, ApJ, 478, L57

123. Hernández-Monteagudo, C., Haiman, Z., Jimenez, R., \& Verde, L. 2007, ApJ, 660, L85

124. Hernández-Monteagudo, C., Haiman, Z., Verde, L. \& Jimenez, R. 2007, ApJ, submitted, arxiv preprint astro-ph:0709.3313

125. Mortonson, M. J., \& Hu, W. 2007, ApJ, submitted, arxiv preprint astro-ph:0705.1132

126. Nemiroff, R. J. 2003, AJ, 125, 2740

127. Oh, S. P. 2001, ApJ, 553, 499

128. Oh, S. P., \& Haiman, Z. 2002, ApJ, 569, 558

129. Oh, S. P., \& Haiman, Z. 2003, MNRAS, 346, 456

130. Oh, S. P., Haiman, Z., \& Rees, M. J. 2001, ApJ, 553, 73

131. Omukai, K., \& Nishi, R. 1999, ApJ, 518, 64

132. O'Shea, B. W., \& Norman, M. L. 2006, ApJ, 648, 31

133. O'Shea, B. W., \& Norman, M. L. 2007, ApJ, submitted, arxiv preprint astroph:0706.4416

134. Page, L. et al. 2007, ApJS, 170, 335

135. Patat, F., Barbon, R., Cappellaro, E., \& Turatto, M. 1994, A\&A, 282, 731 
136. Peebles, P. J. E. 2000, ApJ, 534, 127

137. Press, W. H., \& Schechter, P. L. 1974, ApJ, 181, 425

138. Perlmutter, S., et al. 1999, ApJ, 517, 565

139. Riess, A. G., et al. 1998, AJ, 116, 1009

140. Richardson, D., Branch, D., Casebeer, D., Millard, J., Thomas, R. C., \& Baron, E. 2002, AJ, 123, 745

141. Ricotti, M., Gnedin, N. Y., \& Shull, J. M. 2001, ApJ, 560, 580

142. Ricotti, M., Gnedin, N. Y., \& Shull, J. M. 2002a, ApJ, 575, 33

143. Ricotti, M., Gnedin, N. Y., \& Shull, J. M. 2002b, ApJ, 575, 49

144. Ricotti, M., Ostriker, J. P., \& Gnedin, N. Y. 2005, MNRAS, 357, 207

145. Santos, M. G., Cooray, A., Haiman, Z., Knox, L., Ma, C.-P. 2003, ApJ, 598, 756

146. Santos, M. G., Amblard, A., Pritchard, J., Trac, H., Cen, R., Cooray, A. 2007, ApJ, submitted, arxiv preprint astro-ph:0708.2424

147. Saslaw, W. C., \& Zipoy, D. 1967, Nature, 216, 976

148. Schaerer, D. 2002, A\&A, 382, 28

149. Sellwood, J., \& Kosowsky, A. 2001, in "Gas \& Galaxy Evolution”, eds. Hibbard, Rupen \& van Gorkom, astro-ph/0009074

150. Shapiro, P. R., Giroux, M. L., \& Babul, A. 1994, ApJ, 427, 25

151. Shapiro, P. R., Iliev, I. T., \& Raga, A. C. 2004, MNRAS, 348, 753

152. Shapiro, P. R., \& Kang, H. 1987, ApJ, 318, 32

153. Shapley, A. E., Steidel, C. C., Pettini, M., Adelberger, K. L., \& Erb, D. K. 2006, ApJ, 651,688

154. Sheth, R. K., Mo, H. J., \& Tormen, G. 2001, MNRAS, 323, 1

155. Silk, J., \& Spaans, M. 1997, ApJ, 488, L79

156. Slosar, A., McDonald, P., \& Seljak, U. 2007, New Ast. Review, 51, 327

157. Sokasian, A., Yoshida, N., Abel, T., Hernquist, L., \& Springel, V. 2004, MNRAS, 350, 47

158. Somerville, R. S., Primack, J. R., \& Faber, S. M. 2001, MNRAS, 320, 504

159. Spergel, D. N. et al. 2003, ApJS, 148, 175

160. Spergel, D. N., et al. 2007, ApJS, 170, 377

161. Spergel, D. N., \& Steinhardt, P. J. 2000, PRL, 84, 3760

162. Springel, V. et al. 2005, Nature, 435, 629

163. Stanek, K. Z., et al. 2003, ApJ, 591, L17

164. Stanway, E. R., et al. 2004, ApJ, 607, 704

165. Stanway, E. R., et al. 2007, MNRAS, 376, 727

166. Steidel, C. C., Pettini, M., \& Adelberger, K. L. 2001, 546, 665

167. Strolger, L. et al. 2004, ApJ, 613, 200

168. Susa, H., Uehara, H., Nishi, R., \& Yamada, M. 1998, Prog. Theor. Phys. 100, 63

169. Taniguchi, Y., et al. 2005, PASJ, 57, 165

170. Tasitsiomi, A. 2006, ApJ, 645, 792

171. Tegmark, M., Silk, J., Rees, M. J., Abel, T., \& Blanchard, A. 1997, ApJ, 474, L1

172. Tegmark, M., et al. 2004, Phys. Rev. D, id. 693501

173. Thoul, A. A., \& Weinberg, D. H. 1996, ApJ, 465,608

174. Totani, T. 1997, ApJ, 486, L71

175. Totani, T. 1999, ApJ, 511, 41

176. Tumlinson J., Shull J. M. 2000, ApJ, 528, L65

177. Tumlinson, J., Giroux, M. L., \& Shull, M. J. 2001, ApJ, 550, L1

178. Viel, M., Becker, G. D., Bolton, J. S., Haehnelt, M. G., Rauch, M., \& Sargent, W. L. W. 2007, Phys. Rev. D., submitted, arxiv preprint astro-ph:0709.0131 
179. Volonteri, M., \& Rees, M. J. 2005, ApJ, 633, 624

180. Walter, F., et al. 2003, Nature, 424, 406

181. Walter, F., Carilli, C., Bertoldi, F., Menten, K., Cox, P., Lo, K. Y., Fan, X., \& Strauss, M. A. 2004 ApJ, 615, L17

182. Weymann, R. J., Stern, D., Bunker, A., Spinrad, H., Chaffee, F. H., Thompson, R. I., \& Storrie-Lombardi, L. J. 1998, ApJ, 505, L95

183. Whalen, D., Abel, T., \& Norman, M.L. 2004, ApJ, 610, 14

184. Wijers, R. A. M. J. et al. 1998, MNRAS, 294, L13

185. Wise, J. H., \& Abel, T. 2005, ApJ, 629, 615

186. Wise, J. H., \& Abel, T. 2007, ApJ, 671, 1559

187. Wood, K. \& Loeb, A. 1999, ApJ, 545, 86

188. Wyithe, J. S. B., \& Cen, R. 2007, ApJ, 659, 890

189. Wyithe, J. S. B., \& Loeb, A. 2003, ApJ, 588, L69

190. - 2006, Nature, 441, 322

191. Yang, Y., Zabludoff, A. I., Davé, R., Eisenstein, D. J., Pinto, P. A., Katz, N., Weinberg, D. H., \& Barton, E. J. 2006, ApJ, 640, 539

192. Yoshida, N., Abel, T., Hernquist, L., \& Sugiyama, N. 2003, ApJ, 592, 645

193. Yoshida, N., Omukai, K., Hernquist, L., \& Abel, T. 2006, ApJ, 652, 1

194. Yoshida, N., Omukai, K., \& Hernquist, L. 2008, Science, 321, 669

195. Yoshida, N., Sokasian, A., Hernquist, L., \& Springel, V. 2004, ApJ, 591, 1

196. Thomas, R. M. \& Zaroubi, S. 2007, MNRAS, in press, preprint arXiv:0709.1657

197. Zaroubi, S. \& Silk, J. 2005, MNRAS, 360, L64 